\title{
THE VISHIK-LYUSTERNIK METHOD IN THE MIXED PROBLEM FOR PARABOLIC OPERATORS UNRESOLVED WITH RESPECT TO THE HIGHEST TIME DERIVATIVE
}

\author{
L. R. VOLEVICH
}

To Mark Iosifovich Vishik for his 80th birthday

\begin{abstract}
We consider the mixed problem for parabolic operators unresolved with respect to the highest time derivative with boundary conditions of general type and zero initial conditions. We present an analog of the Shapiro-Lopatinskii condition that allows one to obtain two-sided a priori estimates in specially constructed function spaces. In the case considered in this paper the characteristic equation in the half-space has two groups of roots with different asymptotics. Because of this, the crucial role in the study of the problem is played by the Vishik-Lyusternik method in the form presented by Volevich (2006).
\end{abstract}

\section{INTRODUCTION}

In the well-known paper 3 by Agranovich and Vishik the study of the general mixed problem for the $2 b$-parabolic equation (system) in a domain $G$ with smooth boundary $\partial G$

$$
\begin{aligned}
A\left(y, D_{x}, D_{t}\right) U(y) & =F(y), \quad y=(x, t) \in G \times[0, \infty], \\
B_{j}\left(y^{\prime}, D_{x}, D_{t}\right) U\left(y^{\prime}\right) & =G_{j}\left(y^{\prime}\right), \quad y^{\prime}=\left(x^{\prime}, t\right) \in \partial G \times[0, \infty], \quad j=1, \ldots, m,
\end{aligned}
$$

and, say, zero initial conditions was reduced to the study of the elliptic problem with the large complex parameter obtained by the formal Laplace transform:

$$
\begin{aligned}
& A(y, D, \tau) u(x, \tau)=f(x, \tau), \quad x \in G, \quad \operatorname{Im} \tau \leq 0, \\
& B_{j}\left(y^{\prime}, D, \tau\right) u\left(x^{\prime} \cdot \tau\right)=g_{j}\left(x^{\prime}, \tau\right), \quad x^{\prime} \in \partial G, \quad j=1, \ldots, m .
\end{aligned}
$$

Here

$$
\begin{aligned}
A(y, \xi, \tau)= & A_{2 m}(y, \xi)+A_{2 m-2 b}(y, \xi) \tau+\cdots+A_{0}(y) \tau^{p}, \quad 2 b p=2 m, \\
B_{j}\left(y^{\prime}, \xi, \tau\right)= & B_{j, b_{j}}\left(y^{\prime}, \xi\right)+B_{j, b_{j}-2 b}\left(y^{\prime}, \xi\right) \tau+\cdots+B_{j, 0}\left(y^{\prime}, \xi\right) \tau^{p_{j}}, \\
& 2 b p_{j}=b_{j}, \quad j=1, \ldots, m .
\end{aligned}
$$

We assume that ord $A_{2 m-k}(y, \xi)=2 m-k$ and ord $B_{j, b_{j}-k}\left(y^{\prime}, \xi\right)=b_{j}-k$. Denote by $A_{2 m-k}^{0}(y, \xi)$ and $B_{j, b_{j}-k}^{0}\left(y^{\prime}, \xi\right)$ the leading homogeneous terms of the symbols $A_{2 m-k}(y, \xi)$ and $B_{j, b_{j}-k}\left(y^{\prime}, \xi\right)$ respectively. Replacing $A_{2 m-k}(y, \xi)$ with $A_{2 m-k}^{0}(y, \xi)$ and $B_{j, b_{j}-k}\left(y^{\prime}, \xi\right)$ with $B_{j, b_{j}-k}^{0}\left(y^{\prime}, \xi\right)$ we obtain the leading quasihomogeneous terms of the

†Leonid Romanovich Volevich passed away on April 26, 2007.

2000 Mathematics Subject Classification. Primary 35K40; Secondary 35B25.

The work is supported by the Russian Foundation of Fundamental Research, Grant 06-01-00096. 
symbols (1.3) and (1.4):

$$
\begin{aligned}
A^{0}(y, \xi, \tau) & =A_{2 m}^{0}(y, \xi)+A_{2 m-2 b}^{0}(y, \xi) \tau+\cdots, \\
B_{j}^{0}\left(y^{\prime}, \xi, \tau\right) & =B_{j, b_{j}}^{0}\left(y^{\prime}, \xi\right)+B_{j, b_{j}-2 b}^{0}\left(y^{\prime}, \xi\right) \tau+\cdots .
\end{aligned}
$$

The symbols $A^{0}(x, \xi, \tau)$ and $B_{j}^{0}\left(y^{\prime}, \xi, \tau\right)$ become quasihomogeneous if we assign weight $2 b$ to the variable $\tau$.

By the inner symbol of the problem at a point $y^{0} \in G \times[0, \infty]$ we mean the polynomial

$$
A(\xi, \tau):=A^{0}\left(y^{0}, \xi, \tau\right) .
$$

According to [3], a symbol is called a parameter-elliptic symbol if

$$
A(\xi, \tau) \neq 0, \quad|\xi|+|\tau|>0, \quad \operatorname{Im} \tau \leq 0 .
$$

This condition is equivalent to the two-sided estimate

$$
C^{-1}\left(|\xi|+|\tau|^{1 /(2 b)}\right)^{2 m} \leq|A(\xi, \tau)| \leq C\left(|\xi|+|\tau|^{1 /(2 b)}\right)^{2 m}, \quad \operatorname{Im} \tau \leq 0, \quad \xi \in \mathbb{R}^{n} .
$$

The boundary symbol is defined to be the boundary problem for the ordinary differential equation obtained as follows. Let $x^{0}$ be a point of $\partial G$. Let us choose coordinates $\left(x^{\prime}, z\right)$ so that locally the boundary is given by $z=0$. Let $\left(\xi^{\prime}, \zeta\right)$ be dual variables. Set $B_{j}\left(\xi^{\prime}, \zeta, \tau\right)=B_{j}^{0}\left(x^{0}, \xi^{\prime}, \zeta, \tau\right)$. The problem on the half-line $z \geq 0$ defined by the formulas

$$
\begin{aligned}
A\left(\xi^{\prime}, \frac{d}{i d z}, \tau\right) v(z) & =0, \quad z>0, \quad \operatorname{Im} \tau \leq 0, \\
B_{j}\left(\xi^{\prime}, \frac{d}{i d z}, \tau\right) v(0) & =\phi_{j}, \quad j=1, \ldots, m, \\
|v(z)| & \rightarrow 0, \quad z \rightarrow+\infty,
\end{aligned}
$$

is called the boundary symbol at the point $x^{0}$.

The problem (1.2) is called a parameter-elliptic problem if the following conditions are satisfied:

(I) The inner symbol is parameter-elliptic at each point $x^{0} \in \bar{G}$.

(II) For $\operatorname{Im} \tau \leq 0$ and $\left|\xi^{\prime}\right|+|\tau|>0$ the boundary symbol is invertible at each point $x^{0} \in \partial G$, i.e., the problem (1.6) is uniquely solvable for all $\left(\phi_{1}, \ldots, \phi_{m}\right) \in \mathbb{C}^{m}$.

The theory of parameter-elliptic problems (1.2) is "parallel" to the standard elliptic theory and is, in some sense, simpler since Fredholm operators (with finite-dimensional kernel and cokernel) are replaced by operators that are invertible for large $-\operatorname{Im} \tau$.

The described approach becomes significantly more complicated for problems for $2 b$ elliptic operators unresolved with respect to the highest time derivative. Following 4 we will call such problems $2 b$-pseudoparabolic. The corresponding operator in this case has the form

$$
A\left(y, D_{x}, D_{t}\right)=A_{2 m}\left(y, D_{x}\right)+A_{2 m-2 b}\left(y, D_{x}\right) D_{t}+\cdots+A_{2 \mu}\left(y, D_{x}\right) D_{t}^{p},
$$

where $2 m-2 \mu=2 b p$. It is natural to assume that the boundary operators have the form

$$
B_{j}\left(y^{\prime}, D_{x}, D_{t}\right)=B_{j b_{j}}\left(y^{\prime}, D_{x}\right)+B_{j, b_{j}-2 b}\left(y^{\prime}, D_{x}\right) D_{t}+\cdots+B_{j \beta_{j}}\left(y^{\prime}, D_{x}\right) D_{t}^{p_{j}},
$$

where $b_{j}-\beta_{j}=2 b p_{j}$.

The inner symbol of the operator (1.7) vanishes at $\xi=0$ and cannot satisfy the condition of parameter-ellipticity. Therefore, we replace it with the condition of weak parameter-ellipticity; see [6]:

$$
\begin{gathered}
C^{-1}|\xi|^{2 \mu}\left(|\xi|+|\tau|^{1 /(2 b)}\right)^{2 m-2 \mu} \leq|A(\xi, \tau)| \leq C|\xi|^{2 \mu}\left(|\xi|+|\tau|^{1 /(2 b)}\right)^{2 m} \\
\operatorname{Im} \tau \leq 0, \quad \xi \in \mathbb{R}^{n} .
\end{gathered}
$$


This condition implies that

$$
A(\xi, \tau) \neq 0, \quad|\xi| \neq 0, \quad \operatorname{Im} \tau \leq 0,
$$

and the symbols $A_{2 m}^{0}(\xi)$ and $A_{2 \mu}^{0}(\xi)$ are elliptic.

The problem (1.2) for operators (1.7), (1.8) is called weakly parameter-elliptic if

(A) The inner symbol is weakly parameter-elliptic at each point $x^{0} \in \bar{G}$.

Additionally we will assume that at all boundary points $x^{0} \in \partial G$ the polynomials $A_{2 m}^{0}(\xi)$ and $A_{2 \mu}^{0}(\xi)$ are properly elliptic; i.e., half of the roots $z\left(\xi^{\prime}\right)$ of the equation $A_{2 m}\left(\xi^{\prime}, z\right)=0$ and half of the roots of the equation $A_{2 \mu}\left(\xi^{\prime}, z\right)=0$ are in the upper (lower) half-plane of the complex plane. Let us note that for $n>2$ this condition is satisfied automatically.

(B) For $\left|\xi^{\prime}\right|>0, \operatorname{Im} \tau \leq 0$ the boundary symbol (1.2) is invertible at all points $x^{0} \in \partial G$.

Taking $\tau=0$ in Condition (B) we obtain the following condition:

$\left(\mathrm{C}_{1}\right)$ The following problem on the half-line:

$$
\begin{aligned}
A_{2 m}\left(\xi^{\prime}, \frac{d}{i d z}\right) v(z) & =0, \quad z>0, \\
B_{j b_{j}}\left(\xi^{\prime}, \frac{d}{i d z}\right) v(0) & =\phi_{j}, \quad j=1, \ldots, m, \\
v(z) & \rightarrow 0, \quad t \rightarrow+\infty,
\end{aligned}
$$

is uniquely solvable for $\left|\xi^{\prime}\right|=1$.

In other words, the symbols $A_{2 m}, B_{1 b_{1}}, \ldots, B_{m b_{m}}$ are connected by the standard Shapiro-Lopatinskii conditions.

As opposed to elliptic problems with a parameter, condition (B) (and its consequence, condition $\left(\mathrm{C}_{1}\right)$ ) is not sufficient for the validity of two-sided estimates and should be supplemented by conditions that characterize the behavior of problem (1.6) for large $|\tau|$. To do this, it is convenient to replace the large parameter $\tau$ with the related small parameter $\varepsilon^{2 b}=1 / \tau$. The problem (1.2) takes the following form (if we disregard powers of $\varepsilon$ at functions on the right-hand sides):

$$
\begin{aligned}
\tilde{A}\left(y, D_{x}, \varepsilon\right) v(x) & =f(x), \quad x \in G, \\
\tilde{B}_{j}\left(y^{\prime}, D_{x}, \varepsilon\right) v\left(x^{\prime}\right) & =g_{j}\left(x^{\prime}\right), \quad x^{\prime} \in \partial G, \quad j=1, \ldots, m,
\end{aligned}
$$

where

$$
\tilde{A}(y, \xi, \varepsilon)=\varepsilon^{2 m-2 \mu} A_{2 m}(y, \xi)+\varepsilon^{2 m-2 \mu-2 b} A_{2 m-2 b}(y, \xi)+\cdots+A_{2 \mu}(y, \xi)
$$

and

$$
\tilde{B}_{j}\left(y^{\prime}, \xi, \varepsilon\right)=\varepsilon^{b_{j}-\beta_{j}} B_{j, b_{j}}\left(y^{\prime}, \xi\right)+\varepsilon^{b_{j}-\beta_{j}-2 b} B_{j, b_{j}-2 b}\left(y^{\prime}, \xi\right)+\cdots+B_{j, \beta_{j}}\left(y^{\prime}, \xi\right)
$$

for $j=1, \ldots, m$.

For $\varepsilon=0$ the problem (1.11) turns into the problem for a properly elliptic operator $A_{2 \mu}(y, \xi)$ of order $2 \mu$ with $m>\mu$ boundary conditions corresponding to the operators $B_{1, \beta_{1}}\left(y^{\prime}, D_{x}\right), \ldots, B_{m, \beta_{m}}\left(y^{\prime}, D_{x}\right)$. A detailed analysis of this problem using the VishikLyusternik method [1] was performed in 2]. To use this method the boundary operators were numbered in such a way that

$$
\beta_{1} \leq \beta_{2} \leq \cdots \leq \beta_{m}
$$

In addition, we make the following assumption:

$$
\beta_{\mu}<\beta_{\mu+1} .
$$

With this assumption, following [2] we impose the following condition. 
$\left(\mathrm{C}_{2}\right)$ The problem on the half-line

$$
\begin{aligned}
A_{2 \mu}\left(\xi^{\prime}, \frac{d}{i d z}\right) v(z) & =0, \quad z>0, \\
B_{j \beta_{j}}\left(\xi^{\prime}, \frac{d}{i d z}\right) v(0) & =\phi_{j}, \quad j=1, \ldots, \mu \\
v(z) & \rightarrow 0, \quad t \rightarrow+\infty
\end{aligned}
$$

is uniquely solvable for $\left|\xi^{\prime}\right|=1$.

In other words, the symbols $A_{2 \mu}, B_{1 \beta_{1}}, \ldots, B_{\mu \beta_{\mu}}$ are connected by the standard Shapiro-Lopatinskii conditions.

As for the remaining $m-\mu$ boundary operators, the condition suggested by the VishikLyusternik method takes the following form (see [6]):

$\left(\mathrm{C}_{3}\right)$ The problem on the half-line

$$
\begin{aligned}
A\left(0, \frac{d}{i d z}, \theta\right) v(z) & =0, \quad z>0, \\
B_{j}\left(0, \frac{d}{i d z}, \theta\right) v(0) & =\phi_{j}, \quad j=\mu+1, \ldots, m, \\
v(z) & \rightarrow 0, \quad t \rightarrow+\infty
\end{aligned}
$$

is uniquely solvable for all $\theta \in \mathbb{C}, \operatorname{Im} \theta \leq 0,|\theta|=1$.

The main result of the present paper is that conditions (A), (B), $\left(\mathrm{C}_{1}\right),\left(\mathrm{C}_{2}\right),\left(\mathrm{C}_{3}\right)$ are necessary and sufficient for the existence of two-sided estimates both for the stationary problem (1.2) and for the nonstationary problem (1.1). Let us note that the corresponding estimates for the stationary problem (1.2) with boundary conditions independent of the parameter were obtained in 6 .

The corresponding a priori estimates for solutions of the nonstationary problem in the half-space in function spaces related to $L_{p}, 1<p<\infty$, were obtained in the book [4. In this book the authors considered problems with zero boundary conditions. In this more simple case one does not need to construct boundary spaces, and conditions (A) and (B) suffice. Additional conditions $\left(\mathrm{C}_{2}\right)$ and $\left(\mathrm{C}_{3}\right)$ (necessary in the case of problems with nonhomogeneous boundary conditions) were not used in deriving estimates in [4.

The main goal of this paper is to investigate the role of the Vishik-Lyusternik method in the analysis of the mixed problem for $2 b$-parabolic equations unresolved with respect to the highest time derivative, and to find the relation of this problem with traditional elliptic problems with small parameter. Therefore, we consider here the simplest case where the domain $G$ is a half-space, the main operator and boundary operators are quasihomogeneous and have constant coefficients, and the initial conditions are zero.

Let us note that from the obtained two-sided estimates for constant coefficients one can rather easily deduce estimates for slowly varying coefficients in the half-space, whereas a standard well-developed technique allows one to obtain a priori estimates for bounded domains. The right and left parametrix constructed in [6] for problems with boundary operators without time derivatives can also be constructed in the case under consideration.

More difficult are solvability questions for the problem with a parameter in a bounded domain and for the mixed problem in bounded cylindrical domains. We hope to consider these questions in a separate paper.

M. S. Agranovich read the preliminary version of this paper and made a number of useful comments. I use this opportunity to express to him my deep gratitude. 


\section{WEAKLY PARAMETER-ELLIPTIC POLYNOMIALS}

We will consider the polynomials defined in the Introduction, replacing in these polynomials the parameter $\tau$ with the parameter $\lambda=\tau^{1 /(2 b)}$ running over a certain sector $V$ of the complex plane centered at the origin. The sector corresponding to the case of the half-plane $\{\operatorname{Im} \tau<0\}$ we are interested in is $V:=\left\{\lambda \in \mathbb{C}, \frac{\pi}{(2 b)}<\arg \lambda<\frac{\pi}{b}\right\}$.

The initial definition and its implications were discussed in [5] and [6]. We present, without proofs, some results from these papers, complementing them with more precise results from [2] about proper ellipticity for polynomials with small parameters.

2.1. Consider polynomials of the form

$$
A(\xi, \lambda):=A_{2 m}(\xi)+\lambda A_{2 m-1}(\xi)+\cdots+\lambda^{2 m-2 \mu} A_{2 \mu}(\xi)
$$

in variables $\xi=\left(\xi_{1}, \ldots, \xi_{n}\right) \in \mathbb{R}^{n}$ and $\lambda \in V$. As in the introduction, the degree of the polynomial $A_{2 m-j}(\xi)$ equals $2 m-j$. By $A_{2 m-j}^{0}(\xi)$ we denote the leading homogeneous part of the polynomial $A_{2 m-j}$. Replacing in (2.1) the polynomial $A_{2 m-j}(\xi)$ with $A_{2 m-j}^{0}(\xi)$ we obtain the leading part of $A^{0}(\xi, \lambda)$.

Definition 2.1. The polynomial (2.1) is called weakly parameter-elliptic if for some sector $V \in \mathbb{C}$ the leading part of $A^{0}(\xi, \lambda)$ admits the lower bound

$$
\left|A^{0}(\xi, \lambda)\right| \geq C|\xi|^{2 \mu}(|\lambda|+\varepsilon|\xi|)^{2 m-2 \mu}, \quad \lambda \in V .
$$

Let us note that (2.1) and the definition of $A^{0}(\xi, \lambda)$ show that (2.2) is, in fact, a two-sided estimate.

Proposition 2.2 ([5], see also [2]). A polynomial (2.1) is weakly parameter-elliptic if and only if the following conditions hold:

(i) the polynomial $A_{2 m}(\xi)$ is elliptic,

(ii) the polynomial $A_{2 \mu}(\xi)$ is elliptic,

(iii) $A^{0}(\xi, \lambda) \neq 0,|\xi|>0, \lambda \in V$.

2.2. Let us set $\xi=\left(\xi^{\prime}, \xi_{n}\right), \xi^{\prime}=\left(\xi_{1}, \ldots, \xi_{n-1}\right)$ and consider zeros of the algebraic equation

$$
A^{0}\left(\xi^{\prime}, z, \lambda\right)=0, \quad\left(\xi^{\prime}, \lambda\right) \in \mathbb{R}^{n-1} \times V,
$$

in $z$. In principle, we are interested in zeros in the half-plane $\mathbb{C}_{+}$of the complex plane. However, we start with a discussion of the dependence of all roots of (2.3) on $\lambda$. Since the coefficients of our equation are polynomials in $\lambda$, we can choose $2 m$ continuous branches $z_{j}\left(\xi^{\prime}, \lambda\right), j=1, \ldots, 2 m$. In general, the choice of these branches is not unique. By homogeneity of our equation, we can choose the branches in such a way that they are homogeneous functions of $\left(\xi^{\prime}, \lambda\right)$ of degree 1 .

By $S\left(\xi^{\prime}, \lambda\right)$ and $S_{2 \mu}\left(\xi^{\prime}\right)$ we denote the sets on the complex plane $\mathbb{C}$ that correspond to the roots of (2.3) and of the equation

$$
A_{2 \mu}^{0}\left(\xi^{\prime}, z\right)=0
$$

respectively. Since for $\lambda \neq 0$ equation (2.3) is equivalent to the equation

$$
A_{2 \mu}\left(\xi^{\prime}, z\right)+\lambda^{-1} A_{2 \mu+1}\left(\xi^{\prime}, z\right)+\cdots+\lambda^{2 \mu-2 m} A_{2 m}\left(\xi^{\prime}, z\right)=0
$$

for large $|\lambda|$ there are $2 \mu$ roots of (2.3) close to the roots of (2.4). The main idea used in the description of the remaining roots belongs to Vishik and Lyusternik [1].

Following [1] introduce the polynomial

$$
Q(z, \theta):=\frac{A^{0}(0, z, 1)}{A_{2 \mu}^{0}(0, z)}=\sum_{j=0}^{2 m-2 \mu} q_{j} z^{2 m-2 \mu-j}, \quad q_{j}=\frac{A_{2 m-j}^{0}(0,1, \theta)}{A_{2 \mu}^{0}(0,1)}
$$


where we set $\lambda=|\lambda| \theta$. Denote by $S_{b}(\theta)$ the set on the complex plane $\mathbb{C}$ corresponding to the roots of this polynomial.

If $z_{j}\left(\xi^{\prime}, \lambda\right)$ is a root of (2.3), then, by homogeneity,

$$
z_{j}\left(\xi^{\prime}, \lambda\right)=|\lambda| z_{j}\left(\frac{\xi^{\prime}}{|\lambda|}, \theta\right)
$$

It turns out that there are $2 m-2 \mu$ roots of (2.3) such that

$$
z_{j}\left(\frac{\xi^{\prime}}{|\lambda|}, \theta\right) \rightarrow \zeta_{j}(\theta), \quad|\lambda| \rightarrow \infty
$$

where $\zeta_{j}(\theta)$ is a root of the polynomial (2.5).

Therefore, for sufficiently large $\lambda \in V$ the set $S\left(\xi^{\prime}, \lambda\right)$ breaks into two disjoint subsets

$$
S\left(\xi^{\prime}, \lambda\right)=S^{1}\left(\xi^{\prime}, \lambda\right) \cup S^{2}\left(\xi^{\prime}, \lambda\right),
$$

such that, set-theoretically,

$$
S^{1}\left(\xi^{\prime}, \lambda\right) \rightarrow S_{2 \mu}\left(\xi^{\prime}\right), \quad|\lambda|^{-1} S^{2}\left(\xi^{\prime}, \lambda\right) \rightarrow S_{b}(\theta), \quad \lambda \in V, \quad|\lambda| \rightarrow \infty .
$$

More carefully, we have the following result (see [5] and a more precise result in [2]).

Proposition 2.3. Let a polynomial $A\left(\xi^{\prime}, z, \lambda\right)$ be of the form (2.1) and $\lambda \in V$. Then, after an appropriate numbering of the roots $z_{j}\left(\xi^{\prime}, \lambda\right)$ of the equation (2.3), the roots $z_{j}\left(\xi^{\prime}\right)$ of the equation (2.4), and the roots $\zeta_{j}$ of the equation (2.5), the sets

$$
\begin{aligned}
& S^{1}\left(\xi^{\prime}, \lambda\right)=\left\{z_{1}\left(\xi^{\prime}, \lambda\right), \ldots, z_{2 \mu}\left(\xi^{\prime}, \lambda\right)\right\}, \\
& S^{2}\left(\xi^{\prime}, \lambda\right)=\left\{z_{2 \mu+1}\left(\xi^{\prime}, \lambda\right), \ldots, z_{2 m}\left(\xi^{\prime}, \lambda\right)\right\}
\end{aligned}
$$

have the following properties: for each $\delta>0$ there is $\lambda_{0}>0$ such that for $|\lambda| \geq \lambda_{0}>0$,

(i) the distance between the sets $S^{1}\left(\xi^{\prime}, \lambda\right)$ and $S_{2 \mu}\left(\xi^{\prime}\right)$ is less than $\delta\left|\xi^{\prime}\right|$;

(ii) the distance between the sets $|\lambda|^{-1} S^{2}\left(\xi^{\prime}, \lambda\right)$ and $S_{b}(\theta)$ is less than $\delta\left|\xi^{\prime}\right|^{\kappa}$ for some $0<\kappa \leq 1$.

2.3. In boundary problems, most important are the roots with positive imaginary part. Denote by $m^{ \pm}\left(\xi^{\prime}, \lambda\right)$ the number of roots of equation (2.3) in the open upper (lower) half-plane. If the polynomial (2.1) is weakly parameter-elliptic, equation (2.3) does not have real roots. In this case the numbers $m^{ \pm}\left(\xi^{\prime}, \lambda\right)$ do not depend on $\xi^{\prime}$ and $\lambda$, and we denote them by $m^{ \pm}, m^{+}+m^{-}=2 m$. Since the roots of (2.3) continuously depend on $\lambda$, the numbers $m^{ \pm}$equal the number of roots of the equation $A_{2 m}^{0}\left(\xi^{\prime}, \tau\right)=0$ (corresponding to $\lambda=0$ ) in the upper (lower) half-plane.

Next, by $\mu^{ \pm}$and $q^{ \pm}(\theta)$ we denote the number of the corresponding roots of equations (2.4) and $Q(\zeta, \theta)=0$ respectively.

By ellipticity, the polynomial $A_{2 \mu}^{0}$ has no real roots for $\xi^{\prime} \neq 0$. In this case the numbers $\mu^{ \pm}$do not depend on $\xi^{\prime}$ and $\mu^{+}+\mu^{-}=2 \mu$.

If the condition of weak parameter-ellipticity is satisfied, we set in (2.2) $\xi^{\prime}=0, \xi_{n}=$ $|\lambda| \zeta$. Let us note that

$$
A^{0}(0,|\lambda| \zeta, \lambda)=|\lambda|^{2 m} A_{2 \mu}^{0}(0, \zeta) Q(\zeta, \theta) .
$$

Dividing by $|\lambda|^{2 m} \zeta^{2 \mu}$ we obtain

$$
|Q(\zeta, \theta)|>C(1+|\zeta|)^{2 m-2 \mu}
$$

Therefore, the polynomial $Q(\zeta, \theta)$ does not have real roots, the numbers $q^{ \pm}(\theta)$ do not depend on $\theta$, and $q^{+}+q^{-}=2 m-2 \mu$.

By the condition of weak parameter-ellipticity, the sets of roots considered in Proposition 2.3 break into disjoint subsets lying in the upper (lower) half-plane of the complex 
plane. The roots in the upper (lower) half-plane and the polynomials with such roots will be marked by the index $+(-)$.

All this implies that there exist roots $z_{j}^{+}\left(\xi^{\prime}, \lambda\right), j=1, \ldots, \mu^{+}$that tend to the roots $z_{j}^{+}\left(\xi^{\prime}\right), j=1, \ldots, \mu^{+}$, and the roots $z_{j}^{+}\left(\xi^{\prime}, \lambda\right), j=\mu^{+}+1, \ldots, m^{+}$, such that $z_{j}^{+}\left(\xi^{\prime}, \lambda\right) /|\lambda| \rightarrow \zeta_{j}(\theta)$, as $|\lambda| \rightarrow \infty$. Therefore, we have the equalities

$$
m^{+}=\mu^{+}+q^{+}, \quad m^{-}=\mu^{-}+q^{-} .
$$

Hence, we have proved the following result.

Proposition 2.4. Let the polynomial (2.1) be weakly parameter-elliptic and its roots, as well as the roots of the equation (2.4) and the polynomial (2.5), are numbered according to Proposition 2.3. Denote by $S^{1+}\left(\xi^{\prime}, \lambda\right), S^{2+}\left(\xi^{\prime}, \lambda\right), S_{2 \mu}^{+}\left(\xi^{\prime}\right)$, and $S_{b}^{+}(\theta)$ the corresponding sets of roots with positive imaginary parts. Then for each $\delta>0$ there exists $\lambda_{0}>0$ such that for $|\lambda| \geq \lambda_{0}$,

(i) the distance between the sets $S^{1+}\left(\xi^{\prime}, \lambda\right)$ and $S_{2 \mu}^{+}\left(\xi^{\prime}\right)$ is less than $\delta\left|\xi^{\prime}\right|$,

(ii) the distance between the sets $|\lambda|^{-1} S^{2+}\left(\xi^{\prime}, \lambda\right)$ and $S_{b}^{+}(\theta)$ is less than $\delta\left|\xi^{\prime}\right|^{\kappa}$ for some $\kappa \in(0,1]$.

2.4. In the theory of boundary problems an important role is played by the notion of proper ellipticity.

Definition 2.5. A polynomial (2.1) is called properly weakly parameter-elliptic if

$$
m^{+}=m^{-}=m, \quad \mu^{+}=\mu^{-}=\mu .
$$

The condition of proper ellipticity (2.8) and equality (2.7) implies that

$$
q^{+}=q^{-}=m-\mu .
$$

Remark 2.6. 1. It is well known that equalities (2.8) impose additional restrictions only for $n=2$ since for $n>2$ they are satisfied automatically.

2. The equality $q^{+}=q^{-}=m-\mu$ as introduced in [1] as an additional assumption; there it was called the condition of regularity of degeneration. In 2$]$ it was noted that this condition is a consequence of proper weak parameter-ellipticity.

2.5. The decomposition of the set of roots in equation (2.3) into two groups corresponds to the factorization of the polynomial

$$
A^{0}\left(\xi^{\prime}, z, \lambda\right)=A^{01}\left(\xi^{\prime}, z, \lambda\right) A^{02}\left(\xi^{\prime}, z, \lambda\right) .
$$

According to Proposition 2.4 the polynomials $A^{01}$ and $A^{02}$ admit the factorizations $A^{01}=A^{01+} A^{01-}$ and $A^{02}=A^{02+} A^{02-}$. As a result, we obtain the factorization

$$
A^{0}\left(\xi^{\prime}, z, \lambda\right)=A^{01+}\left(\xi^{\prime}, z, \lambda\right) A^{02+}\left(\xi^{\prime}, z, \lambda\right) A^{0-}\left(\xi^{\prime}, z, \lambda\right) .
$$

Polynomials $A^{01+}$ and $A^{02+}$ can be written in the form

$$
\begin{aligned}
& A^{01+}\left(\xi^{\prime}, z, \lambda\right)=z^{\mu}+\sum_{k=1}^{\mu} a_{k}^{1+}\left(\xi^{\prime}, \lambda\right) z^{\mu-k}, \\
& A^{02+}\left(\xi^{\prime}, z, \lambda\right)=z^{m-m u}+\sum_{k=1}^{m-\mu} a_{k}^{2+}\left(\xi^{\prime}, \lambda\right) z^{m-\mu-k},
\end{aligned}
$$

and the coefficients $a_{k}^{1+}\left(\xi^{\prime}, \lambda\right), a_{k}^{2+}\left(\xi^{\prime}, \lambda\right)$ are homogeneous functions of $\left(\xi^{\prime}, \lambda\right)$ of degrees $\mu-k$ and $m-\mu-k$ respectively.

Proposition 2.7. Let the polynomial (2.1) be weakly parameter-elliptic. Then there exists $d>0$ such that the coefficients of polynomials (2.11) and (2.12) are holomorphic functions of $\lambda$ for $\lambda \in V,|\lambda|>d\left|\xi^{\prime}\right|$. 
L. R. VOLEVICH

Proof. We use standard arguments involving the preparatory Weierstrass theorem (see [10, Chapter II B]) and [11, Proposition 1 in III.1.3]).

1. First of all, note that the coefficients of the polynomial (2.11) are elementary symmetric functions of the roots $z_{j}^{+}\left(\xi^{\prime}, \lambda\right), j=1, \ldots, \mu$. These latter functions can be expressed in terms of the functions

$$
\sum_{k=1}^{\mu}\left(z_{k}^{+}\left(\xi^{\prime}, \lambda\right)\right)^{r}, \quad r=1, \ldots, \mu-1 .
$$

Similarly, the coefficients (2.12) are expressed in terms of the functions

$$
\sum_{k=\mu+1}^{m}\left(z_{k}^{+}\left(\xi^{\prime}, \lambda\right)\right)^{r}, \quad r=\mu+1, \ldots, m .
$$

We will prove that the sums (2.13) and (2.14) are holomorphic for $\lambda \in V,|\lambda|>d\left|\xi^{\prime}\right|$.

2. By homogeneity,

$$
z_{k}^{+}\left(\xi^{\prime}, \lambda\right)=\left|\xi^{\prime}\right| z_{k}^{+}\left(\frac{\xi^{\prime}}{\left|\xi^{\prime}\right|}, \frac{\lambda}{\left|\xi^{\prime}\right|}\right) .
$$

Therefore, it suffices to prove that the functions (2.13) and (2.14) are holomorphic for $\left|\xi^{\prime}\right|=1$ and $\lambda \in V,|\lambda|>d$ for some $d>0$.

3. Let us choose a contour $\gamma_{1}^{+}$that lies in the half-plane $\operatorname{Im} z>0$ and contains all roots of (2.4) with positive imaginary part. According to Propositions 2.3 and 2.4 there exists $d>0$ such that for $\left|\xi^{\prime}\right|=1$ and $|\lambda|>d$ the roots $z_{j}^{+}\left(\xi^{\prime}, \lambda\right), j=1, \ldots, \mu$, lie inside the contour $\gamma_{1}^{+}$, whereas the remaining roots of $A^{0}$ lie at the positive distance from this contour. Then

$$
\sum_{k=1}^{\mu}\left(z_{k}^{+}\left(\xi^{\prime}, \lambda\right)\right)^{r}=\int_{\gamma_{1}^{+}} \frac{z^{r} \partial_{z} A^{0}\left(\xi^{\prime}, z, \lambda\right)}{A^{0}\left(\xi^{\prime}, z, \lambda\right)} d z .
$$

The expression under the integral sign is a rational function of $\lambda$ that has no poles for $|\lambda|>d$. Therefore, (2.13) is holomorphic.

4. To prove that the expressions (2.14) are holomorphic, let us note that the numbers

$$
\zeta_{j}^{+}\left(\xi^{\prime}, \lambda\right):=\frac{z_{j}^{+}\left(\xi^{\prime}, \lambda\right)}{\lambda}, \quad j=\mu+1, \ldots, m,
$$

are the roots of the equation

$$
A^{0}\left(\frac{\xi^{\prime}}{\lambda}, \zeta, 1\right)=0
$$

As before, let us choose a contour $\gamma_{2}^{+}$in the half-plane $\operatorname{Im} z>0$ containing all the roots of the polynomial (2.5) with positive imaginary part. According to Propositions 2.3 and 2.4 there exists $d>0$ such that for $\left|\xi^{\prime}\right|=1,|\lambda|>d$ all the roots of (2.16) lie inside this contour. Then

$$
\lambda^{-r} \sum_{k=\mu+1}^{m}\left(z_{k}^{+}\left(\xi^{\prime}, \lambda\right)\right)^{r}=\sum_{k=\mu+1}^{m}\left(\zeta_{k}^{+}\left(\xi^{\prime}, \lambda\right)\right)^{r}=\int_{\gamma_{2}^{+}} \frac{\zeta^{r} \partial_{\zeta} A^{0}\left(\frac{\xi^{\prime}}{\lambda}, \zeta, 1\right)}{A^{0}\left(\frac{\xi^{\prime}}{\lambda}, \zeta, 1\right)} d \zeta .
$$

This completes the proof. 


\section{THE BOUNDARY SYMBOL OF THE WEAKLY PARAMETER-ELLIPTIC PROBLEM. ESTIMATES FOR A FUNDAMENTAL SYSTEM OF SOLUTIONS}

In this section we study the problem on the half-line

$$
\begin{aligned}
A\left(\xi^{\prime}, D_{z}, \lambda\right) v(z) & =0, \quad z>0, \\
B_{j}\left(\xi^{\prime}, D_{z}, \lambda\right) v(0) & =\phi_{j}, \quad j=1, \ldots, m, \\
v(z) & \rightarrow 0, \quad z \rightarrow+\infty,
\end{aligned}
$$

depending on the parameters $\xi^{\prime}$ and $\lambda$. Here

$$
A(\xi, \lambda):=A_{2 m}(\xi)+\lambda A_{2 m-1}(\xi)+\cdots+\lambda^{2 m-2 \mu} A_{2 \mu}(\xi),
$$

where each polynomial $A_{2 m-k}$ coincides with its leading term, i.e., is a homogeneous function of order $2 m-k$. Similarly,

$$
B_{j}(\xi, \lambda)=B_{j b_{j}}(\xi)+\lambda B_{j, b_{j}-1}(\xi)+\cdots+\lambda^{b_{j}-\beta_{j}} B_{j \beta_{j}}(\xi),
$$

where $B_{j, b_{j}-k}$ are homogeneous functions of degree $b_{j}-k$. As in the Introduction, we will assume that the sequence $\beta_{j}$ is monotone nondecreasing, i.e.,

$$
\beta_{1} \leq \beta_{2} \leq \cdots \leq \beta_{\mu}<\beta_{\mu+1} \leq \cdots \leq \beta_{m} .
$$

Definition 3.1. The problem (3.1) is called weakly parameter-elliptic if the following conditions are satisfied (see the Introduction).

Condition (A). The polynomial (3.2) satisfies the condition of weak parameterellipticity in a sector $V \in \mathbb{C}$ with the vertex at the origin. Moreover, this polynomial satisfies the condition of proper weak parameter-ellipticity.

Condition (B). The problem (3.1) is uniquely solvable for $\left|x i^{\prime}\right|>0$ and $\lambda \in V$.

Condition $\left(\mathrm{C}_{1}\right)$. Operators $A_{2 m}, B_{1 b_{1}}, \ldots, B_{m b_{m}}$ satisfy the Shapiro-Lopatinskii condition.

Condition $\left(\mathrm{C}_{2}\right)$. Operators $A_{2 \mu}, B_{1 \beta_{1}}, \ldots, B_{\mu \beta_{\mu}}$ satisfy the Shapiro-Lopatinskii condition (condition (3.4) is assumed to be satisfied).

Condition $\left(\mathrm{C}_{3}\right)$. Operators $A\left(0, D_{z}, \theta\right), B_{\mu+1}\left(0, D_{z}, \theta\right), \ldots, B_{m}\left(0, D_{z}, \theta\right)$, where $\theta \in V$ and $|\theta|=1$, satisfy the Shapiro-Lopatinskii condition.

Let conditions (A) and (B) be satisfied. Then for $\left|\xi^{\prime}\right|>0$ and $\lambda \in V$ there exist $m$ linearly independent solutions $v_{1}\left(z, \xi^{\prime}, \lambda\right), \ldots, v_{m}\left(z, \xi^{\prime}, \lambda\right)$ of the problem

$$
\begin{aligned}
A\left(\xi^{\prime}, D_{z}, \lambda\right) v_{j}(z) & =0, \quad z>0, \\
B_{k}\left(\xi^{\prime}, D_{z}, \lambda\right) v_{j}(0) & =\delta_{k j}, \quad k=1, \ldots, m, \\
\left|v_{j}(z)\right| & \rightarrow 0, \quad z \rightarrow+\infty ;
\end{aligned}
$$

this system is called a fundamental system of solutions of the problem (3.1). The description of the roots of (2.3) implies that for fixed $\xi^{\prime} \neq 0$ and $\lambda \in V$ solutions of (3.5) exponentially decrease as $z \rightarrow \infty$. Therefore, for any fundamental system of solutions, the integral norms

$$
\begin{gathered}
\left\|D_{z}^{\ell} v_{j}\left(\cdot, \xi^{\prime}, \lambda\right)\right\|_{L_{2}\left(\mathbb{R}^{+}\right)}:=\left(\int_{0}^{+\infty}\left|D_{z}^{\ell} v_{j}\left(z, \xi^{\prime}, \lambda\right)\right|^{2} d z\right)^{1 / 2}<\infty \\
j=1, \ldots, m, \quad \ell=0,1,2, \ldots
\end{gathered}
$$

are bounded. One of the main results of the theory under consideration is the following theorem.

Theorem 3.2. Let conditions $(\mathrm{A}),(\mathrm{B}),\left(\mathrm{C}_{1}\right),\left(\mathrm{C}_{2}\right),\left(\mathrm{C}_{3}\right)$ be satisfied. Then there exists $\lambda_{0}>0$ such that for $|\lambda| \geq \lambda_{0}>0$ the following results hold:

(i) solutions of the problem (3.5) are holomorphic functions of $\lambda \in V$; 
(ii) for integrals (3.6) we have the following estimates:

$$
\begin{aligned}
& \left\|D^{l} v_{j}\left(\cdot, \xi^{\prime}, \lambda\right)\right\|_{L_{2}\left(\mathbb{R}^{+}\right)} \\
& \quad \leq \mathrm{const}\left\{\begin{array}{lll}
\left|\xi^{\prime}\right|^{l-\beta_{j}-1 / 2}\left(|\lambda|+\left|\xi^{\prime}\right|\right)^{\beta_{j}-b_{j}}, & j \leq \mu, \quad l \leq \beta_{\mu+1} ; \\
\left|\xi^{\prime}\right|^{\beta_{\mu+1}-\beta_{j}}\left(|\lambda|+\left|\xi^{\prime}\right|\right)^{l-\beta_{\mu+1}+\beta_{j}-b_{j}-1 / 2}, & j \leq \mu, \quad l>\beta_{\mu+1} ; \\
\left|\xi^{\prime}\right|^{l-\beta_{\mu}-1 / 2}\left(|\lambda+| \xi^{\prime} \mid\right)^{\beta_{\mu}-b_{j}}, & j>\mu, \quad l \leq \beta_{\mu} ; \\
\left(|\lambda|+\left|\xi^{\prime}\right|\right)^{l-b_{j}-1 / 2}, & j>\mu, \quad l>\beta_{\mu} .
\end{array}\right.
\end{aligned}
$$

For a problem with boundary conditions that do not depend on a parameter (so that $\left.b_{j}=\beta_{j}, j=1, \ldots, m\right)$, the estimate (3.7) turns into the estimate (3.7) in [6]. In deducing this estimate the authors of [6] used the representation of solutions in the form suggested by Frank 12. The detailed analysis of this construction will allow us to prove part (i). The proof of part (ii) can also be proved using the scheme in [6], but for brevity we will deduce it from results in [2].

As we have already noted in the introduction, introducing a small parameter $\varepsilon=1 / \lambda$ and setting

$$
v_{j}\left(\cdot, \xi^{\prime}, \lambda\right)=\varepsilon^{b_{j}-\beta_{j}} \tilde{v}_{j}\left(\cdot, \xi^{\prime}, \varepsilon\right),
$$

where $\tilde{v}_{j}\left(\cdot, \xi^{\prime}, \varepsilon\right)$ is the solution of the problem

$$
\begin{aligned}
\tilde{A}\left(\xi^{\prime}, D_{z}, \varepsilon\right) \tilde{v}_{j}\left(z, \xi^{\prime}, \varepsilon\right) & =0, \\
\tilde{B}_{j}\left(\xi^{\prime}, D_{z}, \varepsilon\right) \tilde{v}_{j}\left(0, \xi^{\prime}, \varepsilon\right) & =\delta_{k j}, \quad k=1, \ldots, m, \\
\left|\tilde{v}_{j}\left(z, \xi^{\prime}, \varepsilon\right)\right| & \rightarrow 0, \quad z \rightarrow+\infty,
\end{aligned}
$$

for

$$
\begin{aligned}
\tilde{A}(\xi, \varepsilon) & =\varepsilon^{2 m-2 \mu} A_{2 m}(\xi)+\varepsilon^{2 m-2 \mu-1} A_{2 m-1}+\cdots+A_{2 \mu}(\xi), \\
\tilde{B}_{j}(\xi, \varepsilon) & =\varepsilon^{b_{j}-\beta_{j}} B_{j, b_{j}}(\xi)+\varepsilon^{b_{j}-\beta_{j}-1} B_{j, b_{j}-1}(\xi)+\cdots+B_{j, \beta_{j}}(\xi),
\end{aligned}
$$

we reduce the proof of (3.7) to the estimation of solutions of the problem (3.8).

Under the hypotheses of the theorem, the problem (3.8) is elliptic with a small parameter in the sense of [2]. According to Theorem 2.6 in [2] the following inequality holds:

$$
\begin{aligned}
& \left\|D^{\ell} \tilde{v}_{j}\left(\cdot, \xi^{\prime}, \varepsilon\right)\right\|_{L_{2}\left(\mathbb{R}^{+}\right)} \leq \mathrm{const}\left|\xi^{\prime}\right|^{\ell-\beta_{j}-1 / 2} \\
& \quad \times \begin{cases}\left(1+\varepsilon\left|\xi^{\prime}\right|\right)^{\beta_{j}-b_{j}}, & j \leq \mu, \quad \ell \leq \beta_{\mu+1} ; \\
\left(\varepsilon\left|\xi^{\prime}\right|\right)^{\beta_{\mu+1}-\ell+1 / 2}\left(1+\varepsilon\left|\xi^{\prime}\right|\right)^{\ell-\beta_{\mu+1}+\beta_{j}-b_{j}-1 / 2}, & j \leq \mu, \quad \ell>\beta_{\mu+1} ; \\
\left(\varepsilon\left|\xi^{\prime}\right|\right)^{\beta_{j}-\beta_{\mu}}\left(1+\varepsilon\left|\xi^{\prime}\right|\right)^{-b_{j}+\beta_{\mu}}, & j>\mu, \quad \ell \leq \beta_{\mu} ; \\
\left(\varepsilon\left|\xi^{\prime}\right|\right)^{\beta_{j}-\ell+1 / 2}\left(1+\varepsilon\left|\xi^{\prime}\right|\right)^{\ell-b_{j}-1 / 2}, & j>\mu, \quad \ell>\beta_{\mu} .\end{cases}
\end{aligned}
$$

Replacing in (3.9) $\varepsilon$ with $1 /|\lambda|$ and multiplying the resulting inequalities by $|\lambda|^{\beta_{j}-b_{j}}$, we obtain (3.7).

Now let us prove that solutions of the problem (3.5) are holomorphic. It will be convenient to normalize a solution by setting (as in the case of (3.8))

$$
\tilde{v}_{j}\left(\cdot, \xi^{\prime}, \lambda\right)=\lambda^{\beta_{j}-b_{j}} v_{j}\left(\cdot, \xi^{\prime}, \lambda\right) .
$$

This function is a solution of the problem (3.8) with $\varepsilon=1 / \lambda$ and

$$
\tilde{A}(\xi, \lambda)=\lambda^{2 \mu-2 m} A(\xi, \lambda), \quad \tilde{B}_{j}(\xi, \lambda)=\lambda^{\beta_{j}-b_{j}} B_{j}(\xi, \lambda) .
$$

We use the notation introduced in Subsection 2.5. We need two lemmas. 
Lemma 3.3. Let the problem (3.1) satisfy conditions (A), (B), and $\left(\mathrm{C}_{2}\right)$. Let $\left|\xi^{\prime}\right|=1$. Then there is $\lambda_{0}$ such that for $|\lambda|>\lambda_{0}$ there exist polynomials $\tilde{N}_{j}\left(z, \xi^{\prime}, \lambda\right), j=1, \ldots, \mu$, with coefficients homomorphic in $\lambda$ for $\lambda \in V,|\lambda|>\lambda_{0}$, such that

$$
\frac{1}{2 \pi i} \int_{\gamma_{1}^{+}} \frac{\tilde{B}_{k}\left(\xi^{\prime}, z, \lambda\right) \tilde{N}_{j}\left(z, \xi^{\prime}, \lambda\right)}{\tilde{A}^{1+}\left(\xi^{\prime}, z, \lambda\right)} d z=\delta_{j k}, \quad k=1, \ldots, \mu .
$$

Here (in the notation of Subsection 2.5) the contour $\gamma_{1}^{+}$contains all roots of the polynomial $A_{2 \mu}$ with positive imaginary parts and

$$
\tilde{A}^{1+}\left(\xi^{\prime}, z, \lambda\right)=\prod_{j=1}^{\mu}\left(z-z_{j}^{+}\left(\xi^{\prime}, \lambda\right)\right) .
$$

Proof. To construct the polynomials $\tilde{N}_{j}, j=1, \ldots, \mu$, we use a well-known construction from [13]; see also [2, Lemma 2.1]. Let

$$
\tilde{B}_{j}^{\prime}\left(\xi^{\prime}, z, \lambda\right)=\sum_{k=1}^{\mu} b_{j k}\left(\xi^{\prime}, \lambda\right) z^{k-1}, \quad j=1, \ldots, \mu,
$$

be the remainders of the division of $\tilde{B}_{j}$ by $\tilde{A}^{1+}$. Denote by

$$
D\left(\xi^{\prime}, \lambda\right)=\left(d_{j k}\left(\xi^{\prime}, \lambda\right)\right)_{j, k=1, \ldots, \mu}
$$

the matrix inverse to $\left(b_{j k}\right)$. Then the coefficients of the polynomials $\tilde{N}_{j}$ are linear combinations of products of elements of the matrix $D$ and coefficients of the polynomial $\tilde{A}^{1+}$ (the fact that the latter are holomorphic was proved in Proposition 2.7). It remains to prove that the matrix (3.12) exists and its elements are holomorphic.

To do this we note that for $|\lambda| \rightarrow \infty$ the matrix $\left(b_{j k}\left(\xi^{\prime}, \lambda\right)\right)$ tends to the so-called Lopatinskii matrix $\mathcal{L}\left(\xi^{\prime}\right)$ (see [2, Lemma 2.1]) of the boundary problem associated with the operators $\left\{A_{2 \mu}, B_{1, \beta_{1}}, \ldots, B_{\mu, \beta_{\mu}}\right\}$. According to condition $\left(\mathrm{C}_{2}\right)$, these operators are related by the Shapiro-Lopatinskii condition, which is equivalent to invertibility of the matrix $\mathcal{L}$. Since for large $|\lambda|$ the matrix $\left(b_{j k}\left(\xi^{\prime}, \lambda\right)\right)$ is a small perturbation of the matrix $\mathcal{L}$, it has the required properties.

Similar arguments prove the following result.

Lemma 3.4. Let the problem (3.1) satisfy conditions (A), (B), and $\left(\mathrm{C}_{3}\right)$. Let $\left|\xi^{\prime}\right|=$ 1. Then there is $\lambda_{0}$ such that for $|\lambda|>\lambda_{0}$ there exist polynomials $\tilde{N}_{j}\left(z, \xi^{\prime}, \lambda\right), j=$ $\mu+1, \ldots, m$, whose coefficients are holomorphic in $\lambda$ for $\lambda \in V,|\lambda|>\lambda_{0}$ and satisfy the relations

$$
\frac{1}{2 \pi i} \int_{\gamma_{2}^{+}} \frac{\tilde{B}_{k}\left(\frac{\xi^{\prime}}{\lambda}, \zeta, 1\right) \tilde{N}_{j}\left(\zeta, \xi^{\prime}, \lambda\right)}{\tilde{A}^{2+}\left(\frac{\xi^{\prime}}{\lambda}, \zeta, 1\right)} d \zeta=\delta_{j k}, \quad k=\mu+1, \ldots, m .
$$

Here (in the notation of Subsection 2.5) the contour $\gamma_{2}^{+}$contains all the roots $Q(\zeta)$ with positive imaginary parts

$$
\tilde{A}^{2+}\left(\xi^{\prime}, z, \lambda\right)=\prod_{j=\mu+1}^{m}\left(z-z_{j}^{+}\left(\xi^{\prime}, \lambda\right)\right) .
$$

Following $[6$ we will look for a solution of (3.5) of the following form:

$$
\begin{aligned}
\tilde{v}_{j}\left(z, \xi^{\prime}, \lambda\right)= & \sum_{k=1}^{\mu} \psi_{k}\left(\xi^{\prime}, \lambda\right) \frac{1}{2 \pi i} \int_{\gamma_{1}^{+}} \frac{\tilde{N}_{k}\left(\zeta, \xi^{\prime}, \lambda\right)}{\tilde{A}^{1+}\left(\xi^{\prime}, \zeta, \lambda\right)} e^{i z \zeta} d \zeta \\
& +\sum_{k=\mu+1}^{m} \psi_{k}\left(\xi^{\prime}, \lambda\right) \frac{1}{2 \pi i} \int_{\gamma_{2}^{+}} \frac{\tilde{N}_{k}\left(\zeta, \xi^{\prime}, \lambda\right)}{\tilde{A}^{2+}\left(\frac{\xi^{\prime}}{\lambda}, \zeta, 1\right)} e^{i \lambda z \zeta} d \zeta .
\end{aligned}
$$


To determine the coefficients $\psi_{k}$, we apply to both parts of (3.14) the operators $\tilde{B}_{l}\left(\xi^{\prime}, D_{z}, \lambda\right)$ and set $z=0$. Noting that

$$
\left.\tilde{B}_{l}\left(\xi^{\prime}, D_{z}, \lambda\right) e^{i \lambda \zeta z}\right|_{z=0}=\tilde{B}_{l}\left(\xi^{\prime}, \lambda \zeta, \lambda\right)=\lambda^{\beta_{l}} B_{l}\left(\frac{\xi^{\prime}}{\lambda}, \zeta, 1\right),
$$

we obtain the system

$$
\begin{aligned}
\psi_{l}\left(\xi^{\prime}, \lambda\right)+\sum_{k=\mu+1}^{m} h_{l k}\left(\xi^{\prime}, \lambda\right) \psi_{k}\left(\xi^{\prime}, \lambda\right) & =\delta_{l j}, \quad l=1, \ldots, \mu, \\
\sum_{k=1}^{\mu} h_{l k}\left(\xi^{\prime}, \lambda\right) \psi_{k}\left(\xi^{\prime}, \lambda\right)+\lambda^{\beta_{l}} \psi_{l}\left(\xi^{\prime}, \lambda\right) & =\delta_{l j}, \quad l=\mu+1, \ldots, m,
\end{aligned}
$$

where for $l \leq \mu, k>\mu$ we have

$$
\begin{aligned}
h_{l k}\left(\xi^{\prime}, \lambda\right) & =\frac{1}{2 \pi i} \int_{\gamma_{2}^{+}} \frac{\tilde{B}_{l}\left(\xi^{\prime}, \lambda \zeta, \lambda\right) \tilde{N}_{k}\left(\zeta, \xi^{\prime}, \lambda\right)}{\tilde{A}^{2+}\left(\frac{\xi^{\prime}}{\lambda}, \zeta, 1\right)} d \zeta \\
& =\frac{\lambda^{\beta_{l}}}{2 \pi i} \int_{\gamma_{2}^{+}} \frac{\tilde{B}_{l}\left(\frac{\xi^{\prime}}{\lambda}, \zeta, 1\right) \tilde{N}_{k}\left(\zeta, \xi^{\prime}, \lambda\right)}{\tilde{A}^{2+}\left(\frac{\xi^{\prime}}{\lambda}, \zeta, 1\right)} d \zeta=O\left(\lambda^{\beta_{l}}\right), \quad|\lambda| \rightarrow \infty,
\end{aligned}
$$

and for $l>\mu, k \leq \mu$,

$$
h_{l k}\left(\xi^{\prime}, \lambda\right)=\frac{1}{2 \pi i} \int_{\gamma_{1}^{+}} \frac{\tilde{B}_{l}\left(\xi^{\prime}, \zeta, \lambda\right) \tilde{N}_{k}\left(\zeta, \xi^{\prime}, \lambda\right)}{\tilde{A}^{1+}\left(\xi^{\prime}, \zeta, \lambda\right)} d \zeta=O(1), \quad|\lambda| \rightarrow \infty .
$$

As was shown in [6, Lemma 3.3], see also [2, Lemma 2.7], condition (3.4) implies that for sufficiently large $|\lambda|$ the system is uniquely solvable; i.e., its determinant does not vanish identically. Since the coefficients of the system are holomorphic functions (for sufficiently large $|\lambda|)$, the solution $\psi_{1}\left(\xi^{\prime}, \lambda\right), \ldots, \psi_{m}\left(\xi^{\prime}, \lambda\right)$ is holomorphic as well.

\section{Remarks ABOUt SPACES With NORMS DEPENDING ON A PARAMETER}

In this section we present necessary results about norms that will be used to formulate a priori estimates.

4.1. We start with the case of the entire space $\mathbb{R}^{n}$. By $H^{r, s}=H^{r, s}\left(\mathbb{R}^{n}\right), r \geq s \geq 0$, we denote the set of elements of the Sobolev space $H^{r}$ with the norm

$$
\begin{aligned}
\|(1+ & \left.|D|^{2}\right)^{s / 2}\left(1+|\lambda|^{2}+|D|^{2}\right)^{(r-s) / 2} u ; \mathbb{R}^{n} \| \\
& =\left(\int_{\mathbb{R}^{n}}\left(1+|\xi|^{2}\right)^{s}\left(1+|\lambda|^{2}+|\xi|^{2}\right)^{(r-s)}|\hat{u}(\xi)|^{2} d \xi\right)^{1 / 2},
\end{aligned}
$$

where $\hat{u}(\xi)$ is the Fourier transform of the function $u(x)$.

We call two functions $\Phi_{1}(\xi, \lambda)$ and $\Phi_{2}(\xi, \lambda)$ equivalent and write $\Phi_{1}(\xi, \lambda) \approx \Phi_{2}(\xi, \lambda)$ if there exists a constant $C$ (independent of $\xi$ and $\lambda$ ) such that

$$
C^{-1} \Phi_{1}(\xi, \lambda) \leq \Phi_{2}(\xi, \lambda) \leq C \Phi_{1}(\xi, \lambda) .
$$

It is clear that replacing the function $\left(1+|\xi|^{2}\right)^{s / 2}\left(|\lambda|^{2}+|\xi|^{2}\right)^{(r-s) / 2}$ with an equivalent function we obtain an equivalent norm in $H^{r, s}$.

In the case of nonnegative $r \geq s$, we have

$$
\left(1+|\xi|^{2}\right)^{s / 2}\left(1+|\lambda|^{2}+|\xi|^{2}\right)^{(r-s) / 2} \approx 1+|\lambda|^{r-s}+|\xi|^{s}\left(|\lambda|^{2}+|\xi|^{2}\right)^{(r-s) / 2},
$$

and the norm (4.1) is equivalent to the norm

$$
\|u\|_{r, s}=\left\|u ; \mathbb{R}^{n}\right\|+|\lambda|^{r-s}\left\|u ; \mathbb{R}^{n}\right\|+{ }^{\prime}|| u ; \mathbb{R}^{n} \|_{r, s},
$$


where we set

$$
{ }^{\prime}|| u ; \mathbb{R}^{n}\left\|_{r, s}:=|||D|^{s}\left(|\lambda|^{2}+|D|^{2}\right)^{(r-s) / 2} u ; \mathbb{R}^{n}||=|||D|^{s} u ; \mathbb{R}^{n}\right\|_{r-s, 0} .
$$

Let us note that in the case $|\lambda|>\left|\lambda_{0}\right|>0$ the first term on the right-hand side of (4.2) can be omitted.

We will consider the spaces $H^{r, s}$ for negative $s$ as well. Formally, the norm (4.1) makes sense for all real $s$. However, for $s<0$ we define $H^{r, s}$ as the set of functions (distributions) with finite norm (4.3) (see 7] and a detailed discussion in [2]).

In deriving a priori estimates for integer negative $s$ and $r$ we will use the following equivalent norm for (4.3). As before, we set $\xi=\left(\xi^{\prime}, \xi_{n}\right)$. Then (cf. [2, Lemma 3.1])

$$
|\xi|^{2 s}\left(|\lambda|^{2}+|\xi|^{2}\right)^{(r-s)} \approx \sum_{\ell=0}^{s}\left|\xi^{\prime}\right|^{2 s-2 \ell}\left(|\lambda|^{2}+\left|\xi^{\prime}\right|^{2}\right)^{r-s} \xi_{n}^{2 \ell}+\sum_{\ell=s+1}^{r}\left(|\lambda|^{2}+\left|\xi^{\prime}\right|^{2}\right)^{r-\ell} \xi_{n}^{2 \ell}
$$

Introduce the notation

$$
\Phi_{\rho, \sigma}\left(\xi^{\prime}, \lambda\right)= \begin{cases}\left|\xi^{\prime}\right|^{\sigma}\left(|\lambda|^{2}+\left|\xi^{\prime}\right|^{2}\right)^{(\rho-\sigma) / 2}, & \sigma \geq 0 \\ \left(|\lambda|^{2}+\left|\xi^{\prime}\right|^{2}\right)^{\rho / 2}, & \sigma<0\end{cases}
$$

In this notation relation (4.4) can be rewritten as follows:

$$
|\xi|^{2 s}\left(|\lambda|^{2}+|\xi|^{2}\right)^{(r-s)} \approx \sum_{\ell=0}^{r} \Phi_{r-\ell, s-\ell}^{2}\left(\xi^{\prime}, \lambda\right) \xi_{n}^{2 \ell} .
$$

Then the norm (4.3) for integer negative $r$ and $s$ is equivalent to the norm

$$
\left(\sum_{\ell=0}^{r} \int_{-\infty}^{\infty}\left\|\Phi_{r-\ell, s-\ell}\left(D^{\prime}, \lambda\right) D_{n}^{\ell} u\left(\cdot, x_{n}\right) ; \mathbb{R}^{n-1}\right\|^{2} d x_{n}\right)^{1 / 2} .
$$

Let us note that the expression under the integral sign in (4.6) is equal to

$$
\int_{\mathbb{R}^{n}} \Phi_{r-\ell, s-\ell}^{2}\left(\xi^{\prime}, \lambda\right)\left|D_{n}^{\ell} \hat{u}^{\prime}\left(\xi^{\prime}, x_{n}\right)\right|^{2} d \xi^{\prime} d x_{n},
$$

where $\hat{u}^{\prime}\left(\xi^{\prime}, x_{n}\right)$ denotes the partial Fourier transform of the function $u(x)$ in the variables $x^{\prime}$.

4.2. Now we consider functions defined in the half-space

$$
\mathbb{R}_{+}^{n}=\left\{x=\left(x^{\prime}, x_{n}\right), x^{\prime} \in \mathbb{R}^{n-1}, x_{n} \geq 0\right\} .
$$

According to the general definition of function spaces in a domain we define $H^{r, s}\left(\mathbb{R}_{+}^{n}\right)$ as the quotient space $H^{r, s}\left(\mathbb{R}^{n}\right) / H^{r, s}\left(\mathbb{R}^{n}\right)_{-}$, where $H^{r, s}\left(\mathbb{R}^{n}\right)_{-}$is the subspace of $H^{r, s}\left(\mathbb{R}^{n}\right)$ consisting of all functions (distributions) supported in the half-space $\mathbb{R}_{-}^{n}=\left\{\left(x^{\prime}, x_{n}\right), x_{n}\right.$ $\leq 0\}$. We endow $H^{r, s}\left(\mathbb{R}_{+}^{n}\right)$ with the natural quotient norm

$$
\left\|u ; \mathbb{R}_{+}^{n}\right\|_{r, s}:=\inf _{L u \in T(u)}\left\|L u ; \mathbb{R}^{n}\right\|_{r, s},
$$

where $T(u)$ is the coset of $u$, and $L u$ are various representatives of this coset. Let us note that different representatives of the coset $T(u)$ differ only by the values at $x_{n}<0$, so that for $r \geq 0$ the elements of the quotient space can be identified with functions of $u$ defined in the half-space, and $L u$ are extensions of these functions to the entire space.

Let us note that the norms in $H^{r, s}\left(\mathbb{R}_{+}^{n}\right)$ corresponding to equivalent norms in $H^{r, s}\left(\mathbb{R}^{n}\right)$ are equivalent. In particular, for $s \geq 0$, in (4.7) we can use the norm (4.1). Next, 
according to (4.6) the norm (4.7) is equivalent to the norm

$$
\begin{aligned}
\left|\left[u ; \mathbb{R}_{+}^{n}\right]\right|_{r, s}:= & \left(1+|\lambda|^{r-s}\right)\left\|u ; \mathbb{R}_{+}^{n}\right\| \\
& +\left(\sum_{\ell=0}^{r} \int_{0}^{\infty}\left\|\Phi_{r-\ell, s-\ell}\left(D^{\prime}, \lambda\right) D_{n}^{\ell} u\left(\cdot, x_{n}\right) ; \mathbb{R}^{n-1}\right\|^{2} d x_{n}\right)^{1 / 2} .
\end{aligned}
$$

Let us make one more important remark. In defining the norm in $H^{r, s}\left(\mathbb{R}_{+}^{n}\right)$, it is convenient to replace powers of the differential operators $1+|D|^{2}$ and $|\lambda|^{2}+|D|^{2}$ with powers of the pseudodifferential operators $D_{n}+i \sqrt{1+\left|D^{\prime}\right|^{2}} D_{n}+i \sqrt{|\lambda|^{2}+\left|D^{\prime}\right|^{2}}$. Since the symbols $\xi_{n}+i \sqrt{1+\left|\xi^{\prime}\right|^{2}} \xi_{n}+i \sqrt{|\lambda|^{2}+\left|\xi^{\prime}\right|^{2}}$ of these operators are holomorphic in the half-space $\operatorname{Im} \xi_{n}>0$, they map the space of functions supported at $x_{n}<0$ into itself. Then for $s \geq 0$ the norm (4.7) is equivalent to the norm

$$
\left\|R\left(D_{n}+i \sqrt{1+\left|D^{\prime}\right|^{2}}\right)^{s}\left(D_{n}+i \sqrt{1+|\lambda|^{2}+\left|D^{\prime}\right|^{2}}\right)^{r-s} L u ; \mathbb{R}_{+}^{n}\right\| .
$$

Here $L$ is the operator of extension of functions defined in the half-space $\mathbb{R}_{+}^{n}$ to the entire space $\mathbb{R}^{n}$ and $R$ is the operator of restriction from $\mathbb{R}^{n}$ to $\mathbb{R}_{+}^{n}$.

4.3. Now we discuss the traces of functions in the spaces $H^{r, s}\left(\mathbb{R}^{n}\right)$ and $H^{r, s}\left(\mathbb{R}_{+}^{n}\right)$ on the hyperplanes $x_{n}=0$. Replacing in the norms (4.1) and (4.3) $|D|$ and $|\xi|$ with $\left|D^{\prime}\right|$ and $\left|\xi^{\prime}\right|$ respectively, we define the spaces $H^{r, s}\left(\mathbb{R}^{n-1}\right)$.

Since the space $H^{r, s}\left(\mathbb{R}^{n}\right)$ is a subset of the Sobolev space $H^{r}\left(\mathbb{R}^{n}\right)$, for $r>\ell+1 / 2$ we can define the trace operator $\mathcal{T}_{\ell}: u(x) \rightarrow D_{n}^{\ell} u\left(x^{\prime}, 0\right)$. Let us indicate the norms in which these traces are uniformly bounded for $\lambda \in V$.

Proposition 4.1. For $r>\ell+1 / 2, s \neq \ell+1 / 2$, and $\lambda \in V$ we have (in the notation of (4.5)):

$$
\left\|\Phi_{r-\ell-1 / 2, s-\ell-1 / 2}\left(D^{\prime}, \lambda\right)\left(D_{n}^{\ell} f\right)(\cdot, 0) ; \mathbb{R}^{n-1}\right\| \leq \mathrm{const}\left\|f ; \mathbb{R}^{n}\right\|_{r, s}
$$

with a constant that depends on $r, s$, and $\ell$ but does not depend on $\xi^{\prime}$ and $\lambda$.

Proof. We start with the case $s>\ell+1 / 2$. In this case we prove a slightly stronger inequality

$$
\begin{gathered}
\left\|\left(1+\left|D^{\prime}\right|^{2}\right)^{s-\ell-1 / 2}\left(1+|\lambda|^{2}+\left|D^{\prime}\right|^{2}\right)^{(r-s) / 2}\left(D_{n}^{\ell} f\right)(\cdot, 0) ; \mathbb{R}^{n-}\right\| \\
\leq \text { const }\left\|\left(1+|D|^{2}\right)^{s / 2}\left(1+|\lambda|^{2}+|D|^{2}\right)^{(r-s) / 2} f ; \mathbb{R}^{n}\right\| .
\end{gathered}
$$

The latter inequality is a consequence of a general result presented in [8, Theorem 6.1]. According to this result, if a positive function $\chi(\xi)$ satisfies the condition $\chi(\xi) / \chi(\eta) \leq$ const $(1+|\xi-\eta|)^{M}$ with a positive $M$, then

$$
\left\|\left(\chi_{\ell}^{\prime}\left(D^{\prime}\right) D_{n}^{\ell} f\right)(\cdot, 0) ; \mathbb{R}^{n-1}\right\| \leq \text { const }\left\|\chi(D) f ; \mathbb{R}^{n}\right\|,
$$

where

$$
\chi_{\ell}^{\prime}\left(\xi^{\prime}\right)=\left(\int_{-\infty}^{\infty} \xi_{n}^{2 \ell} \chi^{-2}\left(\xi^{\prime}, \xi_{n}\right) d \xi_{n}\right)^{-1 / 2} .
$$

Of course, we assume that the integral on the right-hand side of (4.13) converges.

Now we substitute in the right-hand side of (4.13) the function

$$
\chi(\xi)=\left(1+|\xi|^{2}\right)^{s / 2}\left(1+|\lambda|^{2}+|\xi|^{2}\right)^{(r-s) / 2} .
$$

Making the change of variables $\xi_{n}=\left(1+\left|\xi^{\prime}\right|^{2}\right)^{1 / 2} t$, we obtain the integral

$$
\left(1+\left|\xi^{\prime}\right|^{2}\right)^{-s+\ell+1 / 2}\left(1+|\lambda|^{2}+\left|\xi^{\prime}\right|^{2}\right)^{-r+s} \int_{-\infty}^{\infty} t^{2 \ell}\left(1+t^{2}\right)^{-s}\left(1+\frac{1+\left|\xi^{\prime}\right|^{2}}{1+|\lambda|^{2}+\left|\xi^{\prime}\right|^{2}} t^{2}\right)^{-r+s} d t
$$


and for each $\lambda$ and $\xi^{\prime}$ the expression under the integral sign is estimated by $t^{2 \ell}\left(1+t^{2}\right)^{-s}$. Since $s>\ell+1 / 2$, the integral converges. Taking

$$
\chi_{\ell}^{\prime}\left(\xi^{\prime}\right)=\left(1+\left|\xi^{\prime}\right|^{2}\right)^{s-\ell-1 / 2}\left(1+|\lambda|^{2}+\left|\xi^{\prime}\right|^{2}\right)^{r-s},
$$

we arrive at 4.11).

Consider the case $0 \leq s \leq \ell+1 / 2$. Since, by assumptions, $s \neq \ell+1 / 2$, we have $0 \leq s<\ell+1 / 2$. Substitute again in the right-hand side of (4.13) the function (4.14). Making the change of variables $\xi_{n}=\left(1+|\lambda|^{2}+\left|\xi^{\prime}\right|^{2}\right)^{1 / 2} t$ we obtain

$$
\left(1+|\lambda|^{2}+\left|\xi^{\prime}\right|^{2}\right)^{-r+\ell+1 / 2} \int_{-\infty}^{\infty} t^{2 \ell}\left(\frac{1+\left|\xi^{\prime}\right|^{2}}{1+|\lambda|^{2}+\left|\xi^{\prime}\right|^{2}}+t^{2}\right)^{-s}\left(1+t^{2}\right)^{-r+s} d t .
$$

For $|t| \leq 1$ the expression under the integral sign can be estimated from above by $|t|^{2 \ell-2 s}$. Since $s<\ell+1 / 2$, the integral converges. For $|t| \geq 1$ the expression under the integral sign is estimated from above by $|t|^{2 \ell-2 r}$. Since $r>\ell+1 / 2$, the integral converges at infinity. Therefore, for $0 \leq s<\ell+1 / 2$ we obtain inequality (4.10).

Now consider the case $s<0$. For $\chi(\xi)$ we take the function

$$
|\xi|^{s}\left(|\lambda|^{2}+|\xi|^{2}\right)^{(r-s) / 2} \text {. }
$$

After the change of variables $\xi_{n}=\left(1+|\lambda|^{2}+\left|\xi^{\prime}\right|^{2}\right)^{1 / 2} t$ in the right-hand side of (4.13) we obtain

$$
\left(1+|\lambda|^{2}+\left|\xi^{\prime}\right|^{2}\right)^{-r+\ell+1 / 2} \int_{-\infty}^{\infty} \frac{t^{2 \ell}}{\left(1+t^{2}\right)^{r}}\left[\frac{\left(1+|\lambda|^{2}+\left|\xi^{\prime}\right|^{2}\right) t^{2}+\left|\xi^{\prime}\right|^{2}}{\left(1+|\lambda|^{2}+\left|\xi^{\prime}\right|^{2}\right) t^{2}+1+|\lambda|^{2}+\left|\xi^{\prime}\right|^{2}}\right]^{|s|} d t .
$$

The expression in brackets does not exceed 1, and we obtain (4.10).

According to Proposition 4.1, define the space $\mathcal{H}^{\rho, \sigma}\left(\mathbb{R}^{n-1}\right)$ of functions $f\left(x^{\prime}\right)$ with the norm

$$
\left\|f, \mathcal{H}^{\rho, \sigma}\left(\mathbb{R}^{n-1}\right)\right\|= \begin{cases}(1+|\lambda|)^{\rho-\sigma}\left\|f, \mathbb{R}^{n-1}\right\|+\left\|\Phi_{\rho, \sigma}\left(D^{\prime}, \lambda\right) f, \mathbb{R}^{n-1}\right\|, & \sigma \geq 0 \\ \left\|\Phi_{\rho, \sigma}\left(D^{\prime}, \lambda\right) f, \mathbb{R}^{n-1}\right\|, & \sigma<0\end{cases}
$$

This definition implies that $\mathcal{H}^{\rho, \sigma}\left(\mathbb{R}^{n-1}\right)$ coincides with the space $H^{\rho, \sigma}\left(\mathbb{R}^{n-1}\right)$ for $\sigma \geq 0$, whereas for $\sigma<0$, it is, in general, an extension of the latter space. According to Proposition 4.1, for $r>\ell+1 / 2$ and $s \neq \ell+1 / 2$ we have the inequality

$$
\left\|\left(D_{n}^{\ell} f\right)(\cdot, 0) ; \mathcal{H}^{r-\ell-1 / 2, s-\ell-1 / 2}\left(\mathbb{R}^{n-1}\right)\right\| \leq c\left\|f ; \mathbb{R}^{n}\right\|_{r, s}, \quad \lambda \in V .
$$

In conclusion we note that the definition of the space $H^{r, s}\left(\mathbb{R}_{+}^{n}\right)$ as the quotient space of the space $H^{r, s}\left(\mathbb{R}^{n}\right)$ implies that for $r>\ell+1 / 2$ and $s \neq \ell+1 / 2$ a function $f(x) \in H^{r, s}\left(\mathbb{R}_{+}^{n}\right)$ has the trace $\left(D_{n}^{\ell} f\right)(\cdot, 0)$ and this trace satisfies the inequality (4.10) with the norm in the right-hand side replaced with $\left\|f ; \mathbb{R}_{+}^{n}\right\|_{r, s}$.

\section{A PRiORI ESTimates IN THE HALF-SPACE} FOR WEAKLY PARAMETER-ELLIPTIC PROBLEMS

In this section, we prove a priori estimates in the half-space for solutions of the problem

$$
\begin{aligned}
A\left(D^{\prime}, D_{n}, \lambda\right) u(x) & =f(x), \quad x_{n}>0, \\
\left(B_{j}\left(D^{\prime}, D_{n}, \lambda\right) u\right)\left(x^{\prime}, 0\right) & =g_{j}\left(x^{\prime}\right), \quad j=1, \ldots, m .
\end{aligned}
$$

We will look for estimates in the spaces $H^{r, s}\left(\mathbb{R}_{+}^{n}\right)$, where $r>s \geq 0$. We will not be interested in upper bounds for $r$ and assume that

$$
r-s \geq 2 m-2 \mu, \quad r-s \geq b_{j}-\beta_{j}, \quad r>b_{j}+\frac{1}{2}, \quad j=1, \ldots, m .
$$


L. R. VOLEVICH

Later we will not mention these conditions explicitly. As for $s$, from [7, 2, 6] it follows that the estimates hold in a relatively narrow range.

To the boundary problem (5.1) one can associate the following continuous operator:

$$
\begin{gathered}
H^{r, s}\left(\mathbb{R}_{+}^{n}\right) \rightarrow H^{r-2 m, s-2 \mu}\left(\mathbb{R}_{+}^{n}\right) \times \prod_{i=1}^{m} \mathcal{H}^{r-b_{j}-1 / 2, s-\beta_{j}-1 / 2}, \\
u(x) \rightarrow A(D, \lambda) u(x),\left(B_{1}(D, \lambda) u\right)\left(x^{\prime}, 0\right), \ldots,\left(B_{m}(D, \lambda) u\right)\left(x^{\prime}, 0\right) .
\end{gathered}
$$

We want to find necessary and sufficient conditions for the existence of the a priori estimate

$$
\begin{aligned}
& \left\|u ; \mathbb{R}_{+}^{n}\right\|_{r, s} \leq \mathrm{const}\left(\left\|A(D, \lambda) u ; \mathbb{R}_{+}^{n}\right\|_{r-2 m, s-2 \mu}\right. \\
& \left.\quad+\sum_{j=1}^{m}\left\|B_{j}(D, \lambda) u ; \mathcal{H}^{r-b_{j}-1 / 2, s-\beta_{j}-1 / 2}\left(\mathbb{R}^{n-1}\right)\right\|+\left(1+|\lambda|^{r-s}\right)\left\|u ; \mathbb{R}_{+}^{n}\right\|\right) .
\end{aligned}
$$

Theorem 5.1. For the problem (5.1) the following statements are equivalent.

(I) Conditions $(\mathrm{A}),(\mathrm{B}),\left(\mathrm{C}_{1}\right),\left(\mathrm{C}_{2}\right),\left(\mathrm{C}_{3}\right)$ are satisfied.

(II) Conditions (A), (B) and inequalities (3.7) are satisfied.

(III) Let $r$ and $s$ be natural numbers satisfying (5.2) and the condition

$$
\beta_{\mu}+\frac{1}{2} \leq s<\beta_{\mu+1}+\frac{1}{2}
$$

Then the estimate (5.4) holds.

In principle, this theorem can be easily deduced from results in [2] in the same way as we have already done in Theorem $3.2($ ii). However, for completeness we indicate how to prove estimates (5.4) directly. The necessity of conditions $(\mathrm{A}),(\mathrm{B}),\left(\mathrm{C}_{1}\right),\left(\mathrm{C}_{2}\right),\left(\mathrm{C}_{3}\right)$ (i.e., $(I I I) \rightarrow(I))$ in the case similar to one considered here is proved in [6], so we will not mention the proof of necessity.

For "homogeneous" norms (4.3) the last term on the right-hand side in (5.4) can be omitted. More precisely, we will prove the following theorem.

Theorem 5.2. Let conditions (A) and (B), and inequalities (3.7) and (5.5) be satisfied. Then

$$
\begin{aligned}
& { }^{\prime}\left\|u ; \mathbb{R}_{+}^{n}\right\|_{r, s} \leq \mathrm{const}\left('\left\|A(D, \lambda) u ; \mathbb{R}_{+}^{n}\right\|_{r-2 m, s-2 \mu}\right. \\
& \left.\quad+\sum_{j=1}^{m}\left\|\Phi_{r-b_{j}-1 / 2, s-\beta_{j}-1 / 2}\left(D^{\prime}, \lambda\right)\left(B_{j}(D, \lambda) u\right)(\cdot, 0) ;\left(\mathbb{R}^{n-1}\right)\right\|\right) .
\end{aligned}
$$

As in [2], we start with estimating solutions of the problem (5.1) in the case $f(x) \equiv 0$. This estimate is based on the following important corollary of Theorem 3.2 (ii).

Proposition 5.3. Let inequalities (3.7) be satisfied and let s satisfy the condition (5.5). Then for solutions of the problem (3.5) we have

$$
\left(\int_{0}^{\infty}\left|D_{n}^{\ell} v_{j}\left(x_{n}, \xi^{\prime}, \lambda\right)\right|^{2} d x_{n}\right)^{1 / 2} \leq \mathrm{const} \frac{\Phi_{r-b_{j}-1 / 2, s-\beta_{j}-1 / 2}\left(\xi^{\prime}, \lambda\right)}{\Phi_{r-\ell, s-\ell}\left(\xi^{\prime}, \lambda\right)}, \quad l=0,1, \ldots
$$

Proof. By Theorem 3.2 it suffices to estimate the right-hand side of (5.7) in terms of the right-hand sides of (3.7). Using definition (4.5), let us calculate the right-hand of (5.7) 
for various $j$ and $\ell$. We have

$$
\begin{aligned}
& \frac{\Phi_{r-b_{j}-1 / 2, s-\beta_{j}-1 / 2}\left(\xi^{\prime}, \lambda\right)}{\Phi_{r-\ell, s-\ell}\left(\xi^{\prime}, \lambda\right)} \\
& \quad= \begin{cases}\left|\xi^{\prime}\right|^{\ell-\beta_{j}-\frac{1}{2}}\left(|\lambda|^{2}+\left|\xi^{\prime}\right|^{2}\right)^{\frac{1}{2}\left(\beta_{j}-b_{j}\right)}, & s-\beta_{j}-\frac{1}{2} \geq 0, \ell \leq s ; \\
\left|\xi^{\prime}\right|^{s-\beta_{j}-\frac{1}{2}}\left(|\lambda|^{2}+\left|\xi^{\prime}\right|^{2}\right)^{\frac{1}{2}\left(\beta_{j}-b_{j}+\ell-s\right)}, & s-\beta_{j}-\frac{1}{2} \geq 0, \ell>s ; \\
\left|\xi^{\prime}\right|^{\ell-s}\left(|\lambda|^{2}+\left|\xi^{\prime}\right|^{2}\right)^{\frac{1}{2}\left(s-b_{j}-\frac{1}{2}\right)}, & s-\beta_{j}-\frac{1}{2}<0, \ell \leq s ; \\
\left(|\lambda|^{2}+\left|\xi^{\prime}\right|^{2}\right)^{\frac{1}{2}\left(\ell-b_{j}-\frac{1}{2}\right)}, & s-\beta_{j}-\frac{1}{2}<0, \ell>s .\end{cases}
\end{aligned}
$$

When comparing the right-hand sides of (5.8) and (3.7), we will not distinguish between $\left(|\lambda|^{2}+\left|\xi^{\prime}\right|^{2}\right)^{\frac{1}{2}}$ and $|\lambda|+\left|\xi^{\prime}\right|$.

Let $s-\beta_{j}-1 / 2 \geq 0$ and $\ell \leq s$. According to (5.5), this implies that $j \leq \mu$ and $\ell \leq \beta_{\mu+1}$. In this case the first line on the right-hand side of (5.8) coincides with the first line on the right-hand side of (3.7).

If $\ell>s$ and $s-\beta_{j}-1 / 2 \geq 0$, it remains to consider the case $\ell>\beta_{\mu+1}$. In this case the ratio of the second line on the right-hand side of (5.8) and the similar line on the right-hand side (3.7) equals

$$
\left(\frac{|\lambda|+\left|\xi^{\prime}\right|}{\left|\xi^{\prime}\right|}\right)^{\beta_{\mu+1}+\frac{1}{2}-s} \geq 1
$$

According to (5.5)), the condition $s-\beta_{j}-1 / 2<0$ implies that $j>\mu$, and the condition $\ell \leq s$ implies that $\ell \leq \beta_{\mu}$. In this case the ratio of the third line on the right-hand side of (5.8) and the similar line on the right-hand side of (3.7) equals

$$
\left(\frac{|\lambda|+\left|\xi^{\prime}\right|}{\left|\xi^{\prime}\right|}\right)^{s-\beta_{\mu}-\frac{1}{2}} \geq 1
$$

Finally, if $s-\beta_{j}-1 / 2<0, \ell>\beta_{\mu+1}$, the fourth lines on the right-hand sides of (5.8) and (3.7) coincide.

The proof of the a priori estimate for the homogeneous problem (5.1) almost literally coincides with the proof of Proposition 4.3 in 2. Let us indicate the main steps.

The norm on the left-hand side of (5.6) can be replaced with the equivalent norm (4.6), reducing this inequality to the family of inequalities for $\ell=0,1, \ldots, r$ :

$$
\begin{aligned}
& \int_{0}^{\infty}\left\|\Phi_{r-\ell, s-\ell}\left(D^{\prime}, \lambda\right) D_{n}^{\ell} u\left(\cdot, x_{n}\right) ; \mathbb{R}^{n-1}\right\|^{2} d x_{n} \\
& \quad \leq \mathrm{const} \sum_{j=1}^{m}\left\|\Phi_{r-b_{j}-1 / 2, s-\beta_{j}-1 / 2}\left(D^{\prime}, \lambda\right)\left(B_{j}(D, \lambda) u\right)(\cdot, 0) ;\left(\mathbb{R}^{n-1}\right)\right\| .
\end{aligned}
$$

Making the partial Fourier transform in the variables $x^{\prime}$ we reduce the proof of (5.9) to estimates of the expressions under the integral sign:

$$
\begin{aligned}
& \Phi_{r-\ell, s-\ell}^{2}\left(\xi^{\prime}, \lambda\right) \int_{0}^{\infty}\left|D_{n}^{\ell} \hat{u}^{\prime}\left(\xi^{\prime}, x_{n}\right)\right|^{2} d x_{n} \\
& \quad \leq \mathrm{const} \sum_{j=1}^{m} \Phi_{r-b_{j}-1 / 2, s-\beta_{j}-1 / 2}^{2}\left(\xi^{\prime}, \lambda\right)\left|g_{j}^{\prime}\left(\xi^{\prime}\right)\right|^{2},
\end{aligned}
$$

where $\hat{u}^{\prime}\left(\xi^{\prime}, x_{n}\right)$ denotes the partial Fourier transform of the function $u(x)$ in the variables $x^{\prime}$. For each fixed $\xi^{\prime}$ the function $\hat{u}^{\prime}\left(\xi^{\prime}, x_{n}\right)$ is a solution of a problem of the form (3.1) and can be represented in the form

$$
\hat{u}^{\prime}\left(\xi^{\prime}, x_{n}\right)=\sum_{j=1}^{m} g_{j}^{\prime}\left(\xi^{\prime}\right) v_{j}\left(x_{n}, \xi^{\prime}, \lambda\right),
$$


L. R. VOLEVICH

where $v_{j}\left(x_{n}, \xi^{\prime}, \lambda\right)$ is a solution of the problem (3.5). Substituting (5.11) in (5.10) and using (5.7) we prove (5.6) for $f(x) \equiv 0$.

Now we pass to the proof of the estimate (5.6) in the general case. It repeats a similar proof in [2].

After the partial Fourier transform we obtain

$$
\begin{aligned}
A\left(\xi^{\prime}, D_{n}, \lambda\right) \hat{u}^{\prime}\left(\xi^{\prime}, x_{n}\right) & =\hat{f}^{\prime}\left(\xi^{\prime}, x_{n}\right), \quad x_{n}>0, \\
B_{j}\left(\xi^{\prime}, D_{n}, \lambda\right) \hat{u}^{\prime}\left(\xi^{\prime}, 0\right) & =\hat{g}_{j}^{\prime}\left(\xi^{\prime}\right), \quad j=1, \ldots, m .
\end{aligned}
$$

Represent $\hat{u}^{\prime}\left(\xi^{\prime}, x_{n}\right)$ in the form

$$
\hat{u}^{\prime}\left(\xi^{\prime}, x_{n}\right)=v\left(\xi^{\prime}, x_{n}\right)+w\left(\xi^{\prime}, x_{n}\right),
$$

where $v\left(\xi^{\prime}, x_{n}\right)$ is a specially selected solution of the equation

$$
A\left(\xi^{\prime}, D_{n}, \lambda\right) v\left(\xi^{\prime}, x_{n}\right)=\hat{f}^{\prime}\left(\xi^{\prime}, x_{n}\right), \quad x_{n}>0 .
$$

Then $w\left(\xi^{\prime}, x_{n}\right)$ is a solution of the problem

$$
\begin{aligned}
& A\left(\xi^{\prime}, D_{n}, \lambda\right) w\left(\xi^{\prime}, x_{n}\right)=0, \quad x_{n}>0, \\
& B_{j}\left(\xi^{\prime}, D_{n}, \lambda\right) w\left(\xi^{\prime}, 0\right)={\hat{g_{j}}}^{\prime}\left(\xi^{\prime}\right)-\chi_{j}\left(\xi^{\prime}\right), \quad j=1, \ldots, m,
\end{aligned}
$$

where

$$
\chi_{j}\left(\xi^{\prime}\right)=B_{j}\left(\xi^{\prime}, D_{n}, \lambda\right) v\left(\xi^{\prime}, 0\right) .
$$

We choose a function $v\left(\xi^{\prime}, x_{n}\right)$ satisfying (5.13) for which the following estimates are satisfied:

$$
\begin{aligned}
& \Phi_{r-\ell, s-\ell}^{2}\left(\xi^{\prime}, \lambda\right) \int_{0}^{\infty}\left|D_{n}^{\ell} v\left(\xi^{\prime}, x_{n}\right)\right|^{2} d x_{n} \\
& \quad \leq \mathrm{const} \int_{0}^{\infty}\left|\left(D_{n}+i\left|\xi^{\prime}\right|\right)^{s-2 \mu}\left(D_{n}+i \sqrt{|\lambda|^{2}+\left|\xi^{\prime}\right|^{2}}\right)^{r-2 m-s+2 \mu} \hat{f}^{\prime}\left(\xi^{\prime}, x_{n}\right)\right|^{2} d x_{n} ;
\end{aligned}
$$

$$
\begin{aligned}
& \Phi_{r-b_{j}-1 / 2, s-\beta_{j}-1 / 2}^{2}\left(\xi^{\prime}, \lambda\right)\left|\chi_{j}^{\prime}\left(\xi^{\prime}\right)\right|^{2} \\
& \quad \leq \mathrm{const} \int_{0}^{\infty}\left|\left(D_{n}+i\left|\xi^{\prime}\right|\right)^{s-2 \mu}\left(D_{n}+i \sqrt{|\lambda|^{2}+\left|\xi^{\prime}\right|^{2}}\right)^{r-2 m-s+2 \mu} \hat{f}^{\prime}\left(\xi^{\prime}, x_{n}\right)\right|^{2} d x_{n} .
\end{aligned}
$$

Applying inequality (5.10) to $w\left(\xi^{\prime}, x_{n}\right)$ and using (5.14) and (5.15), we arrive at the estimate

$$
\begin{aligned}
& \Phi_{r-\ell, s-\ell}^{2}\left(\xi^{\prime}, \lambda\right) \int_{0}^{\infty}\left|D_{n}^{\ell} u\left(\xi^{\prime}, x_{n}\right)\right|^{2} d x_{n} \\
& \leq \text { const } \int_{0}^{\infty}\left|\left(D_{n}+i\left|\xi^{\prime}\right|\right)^{s-2 \mu}\left(D_{n}+i \sqrt{|\lambda|^{2}+\left|\xi^{\prime}\right|^{2}}\right)^{r-2 m-s+2 \mu} \hat{f}^{\prime}\left(\xi^{\prime}, x_{n}\right)\right|^{2} d x_{n} \\
& \quad+\operatorname{const} \sum_{j=1}^{m} \Phi_{r-b_{j}-1 / 2, s-\beta_{j}-1 / 2}^{2}\left(\xi^{\prime}, \lambda\right)\left|g_{j}^{\prime}\left(\xi^{\prime}\right)\right|^{2} .
\end{aligned}
$$

Integrating this inequality in $\xi^{\prime}$ and taking the sum of the left-hand sides in $\ell$, we arrive at the estimate (5.6).

For the function $v\left(\xi^{\prime}, x_{n}\right)$ we take

$$
v\left(\xi^{\prime}, x_{n}\right)=R A^{-1}\left(\xi^{\prime}, D_{n}, \lambda\right) E \hat{f}^{\prime}\left(\xi^{\prime}, x_{n}\right) .
$$

Here $E$ is the operator of extension of functions defined on the half-line to the entire line, $R$ is the operator of restriction to the half-line, and $A^{-1}\left(\xi^{\prime}, D_{n}, \lambda\right)$ is the pseudodifferential 
operator with the symbol $A^{-1}\left(\xi^{\prime}, \xi_{n}, \lambda\right)$. Formula (5.13) follows from the fact that the differential operator $A\left(\xi^{\prime}, D_{n}, \lambda\right)$ commutes with the restriction operator $R$.

Let us pass to the proof of the estimate (5.14). By $\hat{v}(\xi)$ and $\widehat{E f}(\xi)$ we denote the Fourier transform in $x_{n}$ of the functions $v\left(\xi^{\prime}, x_{n}\right)$ and $E \hat{f}^{\prime}\left(\xi^{\prime}, x_{n}\right)$ respectively. Substituting the function (5.16) in the left-hand side of (5.14), replacing the integral over the half-line with the integral over the entire line, and using the Parseval formula, we estimate the left-hand side of (5.14) in terms of

$$
\int_{-\infty}^{+\infty}\left[\left(|\lambda|^{2}+\left|\xi^{\prime}\right|^{2}\right)^{r-s}\left|\xi^{\prime}\right|^{2(s-\ell)}\left|A^{-1}(\xi, \lambda) \xi_{n}^{\ell}\right|^{2}\right]|\widehat{E f}(\xi)|^{2} d \xi_{n}
$$

for $s \geq \ell$ and in terms of

$$
\int_{-\infty}^{+\infty}\left[\left(|\lambda|^{2}+\left|\xi^{\prime}\right|^{2}\right)^{r-\ell}\left|A^{-1}(\xi, \lambda) \xi_{n}^{\ell}\right|^{2}\right]|\widehat{E f}(\xi)|^{2} d \xi_{n}
$$

for $s \leq \ell$.

In the first case the expression in brackets under the integral sign does not exceed

$$
\begin{aligned}
& \text { const }\left(|\lambda|^{2}+\left|\xi^{\prime}\right|^{2}\right)^{r-s}\left|\xi^{\prime}\right|^{2(s-\ell)}\left|\xi_{n}\right|^{2 \ell}|\xi|^{-4 \mu}\left(|\lambda|^{2}+|\xi|^{2}\right)^{-2 m+2 \mu} \\
& \quad \leq \mathrm{const}\left(|\lambda|^{2}+|\xi|^{2}\right)^{r-s-2 m+2 \mu}|\xi|^{2(s-2 \mu)} \\
& \quad \leq \mathrm{const}\left|\left(\xi_{n}+i \sqrt{|\lambda|^{2}+\left|\xi^{\prime}\right|^{2}}\right)^{2(r-2 m-s+2 \mu)}\left(\xi_{n}+i\left|\xi^{\prime}\right|\right)^{2(s-2 \mu)}\right| .
\end{aligned}
$$

Let us substitute this estimate in (5.17). We assume that the extension operator $E$ is chosen in such a way that

$$
\int_{-\infty}^{+\infty}\left|D_{n}^{\ell} \widehat{E f}(\xi)\right|^{2} d \xi_{n}=\int_{-\infty}^{+\infty}\left|D_{n}^{\ell} E \hat{f}^{\prime}\left(\xi^{\prime}, x_{n}\right)\right|^{2} d x_{n} \leq \mathrm{const} \int_{0}^{\infty}\left|D_{n}^{\ell} \hat{f}^{\prime}\left(\xi^{\prime}, x_{n}\right)\right|^{2} d x_{n} .
$$

This can be achieved by choosing the Hestenes extension operator (see 2 for more details). Inequality (5.14) for $s \geq \ell$ is proved.

For $\ell>s$ the expression in brackets under the integral sign in (5.18) does not exceed

$$
\text { const }\left(|\lambda|^{2}+|\xi|^{2}\right)^{r-s-2 m+2 \mu}\left(\left(|\lambda|^{2}+|\xi|^{2}\right)^{s-\ell} \xi_{n}^{2 \ell}|\xi|^{-4 \mu}\right) \text {. }
$$

The expression in parentheses can be estimated by

$$
\text { const } \xi_{n}^{2 \ell}|\xi|^{2(s-\ell-2 \mu)} \leq \text { const }|\xi|^{2(s-2 \mu)} .
$$

As before, this implies the estimate (5.14).

Now we pass to the proof of inequality (5.15). Let us note that

$$
\chi_{j}\left(\xi^{\prime}\right)=\int_{-\infty}^{+\infty} \frac{B_{j}(\xi, \lambda)}{A(\xi, \lambda)} \widehat{E f}(\xi) d \xi_{n}:=\int_{-\infty}^{+\infty} H_{j}(\xi, \lambda) F(\xi, \lambda) d \xi_{n},
$$

where we set

$$
\begin{aligned}
F(\xi, \lambda) & =\left(\xi_{n}+i \sqrt{|\lambda|^{2}+\left|\xi^{\prime}\right|^{2}}\right)^{r-2 m-s+2 \mu}\left(\xi_{n}+i\left|\xi^{\prime}\right|\right)^{s-2 \mu} \widehat{E} f(\xi), \\
H_{j}(\xi, \lambda) & =B_{j}(\xi, \lambda) A^{-1}(\xi, \lambda)\left(\xi_{n}-i \sqrt{|\lambda|^{2}+\left|\xi^{\prime}\right|^{2}}\right)^{-r+2 m+s-2 \mu}\left(\xi_{n}-i\left|\xi^{\prime}\right|\right)^{-s+2 \mu} .
\end{aligned}
$$

By the Schwarz inequality,

$$
\left|\chi_{j}\left(\xi^{\prime}\right)\right|^{2} \leq \int_{-\infty}^{+\infty}\left|H_{j}(\xi, \lambda)\right|^{2} d \xi_{n} \int_{-\infty}^{+\infty}|F(\xi, \lambda)|^{2} d \xi_{n},
$$

and the proof of (5.15) is reduced to the proof of the inequality

$$
\Phi_{r-b_{j}-1 / 2, s-\beta_{j}-1 / 2}^{2}\left(\xi^{\prime}, \lambda\right) \int_{-\infty}^{+\infty}\left|H_{j}(\xi, \lambda)\right|^{2} d \xi_{n} \leq \mathrm{const},
$$

where the right-hand side does not depend on $\xi^{\prime}$ and $\lambda$. 
The function $\left|H_{j}(\xi, \lambda)\right|^{2}$ admits the estimate

$$
\left|H_{j}(\xi, \lambda)\right|^{2} \leq \operatorname{const}\left(|\lambda|^{2}+|\xi|^{2}\right)^{-r+s-b_{j}+\beta_{j}}|\xi|^{-2 s+2 \beta_{j}} .
$$

Therefore, we have to prove that

$$
\Phi_{r-b_{j}-1 / 2, s-\beta_{j}-1 / 2}^{2}\left(\xi^{\prime}, \lambda\right) \int_{-\infty}^{+\infty}\left(|\lambda|^{2}+|\xi|^{2}\right)^{-r+s-b_{j}+\beta_{j}}|\xi|^{-2 s+2 \beta_{j}} d \xi_{n} \leq \text { const } .
$$

First we consider the case $s-\beta_{j}-1 / 2>0$. Then the left-hand side of (5.19) takes the form

$$
\left(|\lambda|^{2}+\left|\xi^{\prime}\right|^{2}\right)^{r-s-b_{j}+\beta_{j}}\left|\xi^{\prime}\right|^{2 s-2 \beta_{j}-1} \int_{-\infty}^{+\infty}\left(|\lambda|^{2}+|\xi|^{2}\right)^{-r+s-b_{j}+\beta_{j}}|\xi|^{-2 s+2 \beta_{j}} d \xi_{n} .
$$

Recalling that according to (5.2) we have $r-s-b_{j}+\beta_{j} \geq 0$, we can estimate our expression by

$$
\left|\xi^{\prime}\right|^{2 s-2 \beta_{j}-1} \int_{-\infty}^{+\infty}\left(\left|\xi^{\prime}\right|^{2}+\xi_{n}^{2}\right)^{-s+\beta_{j}} d \xi_{n}=\text { const }
$$

(We used the change of variable $\xi_{n}=\left|\xi^{\prime}\right| t$ under the integral sign.)

In the case $s-\beta_{j}-1 / 2 \leq 0$ the left-hand side of (5.19) takes the form

$$
\left(|\lambda|^{2}+\left|\xi^{\prime}\right|^{2}\right)^{r-b_{j}-1 / 2} \int_{-\infty}^{+\infty}\left(|\lambda|^{2}+|\xi|^{2}\right)^{-r+s-b_{j}+\beta_{j}}|\xi|^{-2 s+2 \beta_{j}} d \xi_{n} .
$$

Similarly to the analysis of (4.14), we make the change of variables $\xi_{n}=\left(|\lambda|^{2}+\left|\xi^{\prime}\right|^{2}\right)^{1 / 2} t$ and obtain

$$
\int_{-\infty}^{\infty} \frac{\left(|\lambda|^{2}+\left|\xi^{\prime}\right|^{2}\right)^{\beta_{j}-s}}{\left(1+t^{2}\right)^{r-s+\beta_{j}-b_{j}}\left(\left(|\lambda|^{2}+\left|\xi^{\prime}\right|^{2}\right) t^{2}+\left|\xi^{\prime}\right|^{2}\right)^{\beta_{j}-s}} d t
$$

Since $s$ and $\beta_{j}$ are integers, the inequality $\beta_{j}-s \geq-1 / 2$ implies that $\beta_{j}-s \geq 0$. But then the latter integral does not exceed

$$
\int_{-\infty}^{\infty}\left(1+t^{2}\right)^{r-s+\beta_{j}-b_{j}} d t=\text { const }
$$

(We used inequalities (5.2).)

\section{Remarks about the spaces of functions in $\mathbb{R}_{x, t}^{n+1}$}

6.1. To pass to the nonstationary problem we will need spaces of functions in the vari-

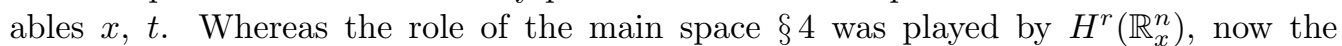
main space is the space $H^{r, \frac{r}{2 b}}\left(\mathbb{R}_{x, t}^{n+1}\right)$ whose elements are smooth of order $r$ in $x$ and of order $\frac{r}{2 b}$ in $t$. As a norm of a function $f$ in this space one can take, for example, $\left\|\left(i D_{t}+1+\left|D_{x}\right|^{2 b}\right)^{\frac{r}{2 b}} f\right\|$.

By $H^{r, \frac{r}{2 b}, s}\left(\mathbb{R}^{n+1}\right), 0 \leq s \leq r$, we denote the space of functions with finite norm

$$
\begin{aligned}
\| & \left(1+\left|D_{x}\right|^{2}\right)^{s / 2}\left(i D_{t}+1+\left|D_{x}\right|^{2 b}\right)^{(r-s) /(2 b)} f \| \\
& =\left(\int_{\mathbb{R}^{n+1}}\left(1+|\xi|^{2}\right)^{s}\left(|\sigma|^{2}+\left(1+\xi^{2 b}\right)^{2}\right)^{(r-s) /(2 b)}|\hat{u}(\xi, \sigma)|^{2} d \xi d \sigma\right)^{1 / 2} .
\end{aligned}
$$

Since, by assumption, $s$ is nonnegative, this norm is equivalent to the norm

$$
\begin{aligned}
& \left\|f, \mathbb{R}^{n+1}\right\|^{r, \frac{r}{2 b}, s}=\left\|\left(i D_{t}+1\right)^{(r-s) /(2 b)} f\right\|+{ }^{\prime}\left\|f, \mathbb{R}^{n+1}\right\|^{r, \frac{r}{2 b}, s}, \\
& '\left\|f, \mathbb{R}^{n+1}\right\|^{r, \frac{r}{2 b}, s}=\left\|\left|D_{x}\right|^{s}\left(i D_{t}+1+\left|D_{x}\right|^{2 b}\right)^{(r-s) /(2 b)} f\right\| .
\end{aligned}
$$

Elements of the space $H^{r, \frac{r}{2 b}, s}\left(\mathbb{R}^{n+1}\right)$ for nonnegative $s$ have finite norm (6.1), whereas for $s<0$ we will use the norm (6.2). 
By $H_{[\gamma]}^{r, \frac{r}{2 b}}\left(\mathbb{R}^{n+1}\right)$, respectively $H_{[\gamma]}^{r, \frac{r}{2 b}, s}\left(\mathbb{R}^{n+1}\right)$, we denote the space of functions that lie in $H^{r, \frac{r}{2 b}}\left(\mathbb{R}^{n+1}\right)$, respectively in $H^{r, \frac{r}{2 b}, s}\left(\mathbb{R}^{n+1}\right)$, after multiplication by the weight $e^{\gamma t}$. For the norms we can take $\left\|e^{\gamma t} f, \mathbb{R}^{n+1}\right\|^{r, \frac{r}{2 b}}$ and $\left\|e^{\gamma t} f, \mathbb{R}^{n+1}\right\|^{r, \frac{r}{2 b}, s}$ respectively. However, we will use a more convenient equivalent (for fixed $\gamma$ ) norm defined as follows. We will assume that $\gamma<0$. Setting $\|g\|_{[\gamma]}=\left\|e^{\gamma t} g\right\|$ we denote

$$
\begin{aligned}
& \left\|f, \mathbb{R}^{n+1}\right\|_{[\gamma]}^{r, \frac{r}{2 b}, s}:=\left\|\left(i D_{t}+1\right)^{(r-s) /(2 b)} f\right\|_{[\gamma]}+{ }^{\prime}\left\|f, \mathbb{R}^{n+1}\right\|_{[\gamma]}^{r, \frac{r}{2 b}, s}, \\
& { }^{\prime}\left\|f, \mathbb{R}^{n+1}\right\|_{[\gamma]}^{r, \frac{r}{2 b}, s}=\left\|\left|D_{x}\right|^{s}\left(i D_{t}+1+\left|D_{x}\right|^{2 b}\right)^{(r-s) /(2 b)} f\right\|_{[\gamma]} .
\end{aligned}
$$

The norm (6.3) is equal to

$$
\left\|\left(i D_{t}+1+|\gamma|\right)^{(r-s) /(2 b)} e^{\gamma t} f, \mathbb{R}^{n+1}\right\|+\left\|\left|D_{x}\right|^{s}\left(i D_{t}+1+|\gamma|+\left|D_{x}\right|^{2 b}\right)^{(r-s) /(2 b)} e^{\gamma t} f\right\| .
$$

6.2. By $H_{[\gamma]+}^{r, \frac{r}{2 b}}\left(\mathbb{R}^{n+1}\right)$ and $H_{[\gamma]+}^{r, \frac{r}{2 b}, s}\left(\mathbb{R}^{n+1}\right)$ we denote the subspaces of the corresponding spaces consisting of the functions supported in the half-space $t \geq 0$.

Theorem 6.1. A function $f(x, t)$ lies in the space $H_{[\gamma]+}^{r, \frac{r}{2 b}, s}\left(\mathbb{R}^{n+1}\right)$ if and only if $f \in$ $H_{[\rho]}^{r, \frac{r}{2 b}, s}\left(\mathbb{R}^{n+1}\right)$ for all $\rho<\gamma$, and

$$
\sup _{\rho<\gamma}\left\|f, \mathbb{R}^{n+1}\right\|_{[\rho]}^{r, \frac{r}{2 b}, s}=\left\|f, \mathbb{R}^{n+1}\right\|_{[\gamma]}^{r, \frac{r}{2 b}, s} .
$$

Theorem 6.2 (Paley-Wiener). A function $f(x, t)$ belongs to the space $H_{[\gamma]+}^{r, \frac{r}{2 b}, s}\left(\mathbb{R}^{n+1}\right)$ if and only if its Fourier-Laplace transform $\hat{f}(\xi, \tau)$ is a holomorphic function of $\tau$ for $\operatorname{Im} \tau<\gamma$ and, for almost all $\xi \in \mathbb{R}^{n}$, the integral

$$
\left(\int_{\mathbb{R}^{n}} \int_{\operatorname{Im} \tau=\rho}\left(1+|\xi|^{2}\right)^{s}\left(1+|\tau|^{2}+|\xi|^{4 b}\right)^{(r-s) /(2 b)}|\hat{u}(\xi, \tau)|^{2} d \xi d \tau\right)^{1 / 2}
$$

is uniformly bounded for $\rho<\gamma$.

In principle, these theorems are well known; see [3]. The above formulations are taken from [11, 1.1.6]. A detailed exposition can be found in [14, Chapter 2, $\S 1$ ].

By $H^{r, \frac{r}{2 b}, s[\gamma]+}\left(\mathbb{R}^{n} \times \mathbb{C}\right)$ we denote the space consisting of functions $f(x, \tau), x \in \mathbb{R}^{n}$, $\tau \in \mathbb{C}$, that are holomorphic in $\tau$ for $\operatorname{Im} \tau<\gamma$ and have finite norm

$$
\begin{aligned}
\left\|f, \mathbb{R}^{n} \times \mathbb{C}\right\|^{r, \frac{r}{2 b}, s[\gamma]+}= & \sup _{\operatorname{Im} \tau<\gamma}\left(\left\|(1+i \tau)^{(r-s) /(2 b)} f(\cdot, \tau)\right\|\right. \\
& \left.+\left\|\left|D_{x}\right|^{s}\left(i \tau+1+\left|D_{x}\right|^{2 b}\right)^{(r-s) /(2 b)} f(\cdot, \tau)\right\|\right) .
\end{aligned}
$$

The above results imply the following theorem.

Theorem 6.3. The partial Fourier-Laplace transform

$$
f(x, t) \Longrightarrow \tilde{f}(x, \tau)=(2 \pi)^{-1 / 2} \int_{0}^{\infty} e^{i t \tau} f(x, t) d t
$$

determines an isomorphism between the spaces $H_{[\gamma]+}^{r, \frac{r}{2 b}, s}\left(\mathbb{R}^{n+1}\right)$ and $H^{r, \frac{r}{2 b}, s[\gamma]+}\left(\mathbb{R}^{n} \times \mathbb{C}\right)$.

Indeed, according to Theorem 6.2 the transform (6.6) yields a function that is holomorphic in $\tau$ for $\operatorname{Im} \tau<\gamma$. Replacing in the definition of the norm (6.1), (6.2) the operator $i D_{t}$ with $i \tau$, we obtain the desired result.

Remark 6.4. If we replace in the norm (6.5) the parameter $\tau$ with $\lambda=(i \tau)^{1 /(2 b)}$, then for $\operatorname{Im} \tau=0$ we obtain a norm equivalent to the norms (4.1)-(4.3). For $V$ we have to take the sector $\left\{\lambda \in \mathbb{C}, \frac{3 \pi}{4 b}<\arg \lambda<\frac{5 \pi}{4 b}\right\}$. Therefore, elements of the spaces $H^{r, \frac{r}{2 b}}, s[\gamma]+\left(\mathbb{R}^{n} \times \mathbb{C}\right)$ belong to the spaces studied in $\S$. 
6.3. In studying the spaces of functions defined in the half-space

$$
\mathbb{R}_{+}^{n+1}=\left\{x=\left(x^{\prime}, x_{n}, t\right), x^{\prime} \in \mathbb{R}^{n-1}, x_{n} \geq 0, t \in \mathbb{R}\right\},
$$

we will follow $\S$ by defining $H_{[\gamma]}^{r, \frac{r}{2 b}, s}\left(\mathbb{R}_{+}^{n+1}\right)$ as the quotient space

$$
H_{[\gamma]}^{r, \frac{r}{2 b}, s}\left(\mathbb{R}^{n+1}\right) / H_{[\gamma]}^{r, \frac{r}{2 b}, s}\left(\mathbb{R}^{n+1}\right)_{-},
$$

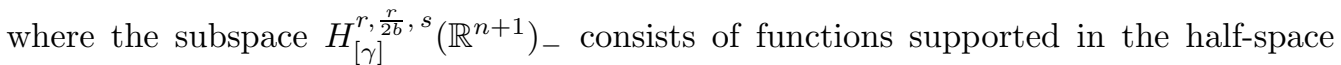
$\mathbb{R}_{-}^{n+1}=\left\{\left(x^{\prime}, x_{n}, t\right), x_{n} \leq 0\right\}$. The space $H_{[\gamma]}^{r, \frac{r}{2 b}, s}\left(\mathbb{R}_{+}^{n+1}\right)$ is endowed with the natural quotient norm.

It is convenient to define the norm in $H_{[\gamma]}^{r, \frac{r}{2 b}, s}\left(\mathbb{R}_{+}^{n+1}\right)$ using pseudodifferential operators with symbols that are holomorphic for $\operatorname{Im} \xi_{n}>0$. These operators leave invariant the space of functions supported in the half-space $x_{n}<0$. Then the initial quotient norm becomes equivalent to the norm

$$
\left\|e^{\gamma t} R\left(D_{n}+i \sqrt{1+\left|D^{\prime}\right|^{2}}\right)^{s}\left(D_{n}+i\left(i D_{t}+\left|D^{\prime}\right|^{2 b}\right)^{\frac{1}{(2 b)}}\right)^{r-s} L f ; \mathbb{R}_{+}^{n+1}\right\| .
$$

Here $L$ is the operator of extension of functions defined on $\mathbb{R}_{+}^{n+1}$ to the entire space $\mathbb{R}^{n+1}$ and $R$ is the operator of restriction of functions on $\mathbb{R}^{n+1}$ to $\mathbb{R}_{+}^{n+1}$.

6.4. We will also need the spaces $H^{r, \frac{r}{2 b}, s}\left(\mathbb{R}^{n}\right)$ of functions defined on the hyperplane $x_{n}=0$. Replacing in (6.2) $D_{x}$ with $D_{x^{\prime}}$ we define the norm in this space. Next we can naturally define the spaces $H_{[\gamma]}^{r, \frac{r}{2 b}, s}\left(\mathbb{R}^{n}\right)$ and $H_{[\gamma]+}^{r, \frac{r}{2 b}, s}\left(\mathbb{R}^{n}\right)$. Since the space $H^{r, \frac{r}{2 b}, s}\left(\mathbb{R}^{n+1}\right)$ is a subset of the space $H^{r, \frac{r}{2 b}}\left(\mathbb{R}^{n+1}\right)$, for $r>\ell+1 / 2$ we can define the trace operator $\mathcal{T}_{\ell}: u(x, t) \rightarrow D_{n}^{\ell} u\left(x^{\prime}, 0, t\right)$.

To estimate the norm of the trace operator we define, similarly to (4.5), the functions

$$
\Psi_{\rho, \theta}\left(\xi^{\prime}, \tau\right)= \begin{cases}\left|\xi^{\prime}\right|^{\theta}\left(i \tau+1+\left|\xi^{\prime}\right|^{2 b}\right)^{(\rho-\theta) /(2 b)}, & \theta \geq 0, \\ \left(i \tau+1+\left|\xi^{\prime}\right|^{2 b}\right)^{\rho /(2 b)}, & \theta<0 .\end{cases}
$$

Repeating arguments in $\S 4$ we can prove the following result.

Proposition 6.5. For $r>\ell+1 / 2$ and $s \neq \ell+1 / 2$ we have the inequality

$$
\left\|\Psi_{r-\ell-1 / 2, s-\ell-1 / 2}\left(D_{x^{\prime}}, D_{t}\right)\left(D_{n}^{\ell} f\right)(\cdot, 0, \cdot) ; \mathbb{R}^{n}\right\|_{[\gamma]} \leq \text { const }\left\|f ; \mathbb{R}^{n+1}\right\|_{[\gamma]}^{r, s} .
$$

Using the notation (6.8) we define the space $\mathcal{H}^{\rho, \theta}\left(\mathbb{R}^{n}\right)_{[\gamma]}$ of functions $f\left(x^{\prime}, t\right)$ with the norm

$$
\left\|f, \mathcal{H}^{\rho, \theta}\left(\mathbb{R}^{n}\right)\right\|_{[\gamma]}= \begin{cases}\left\|\left(i D_{t}+1\right)^{(r-s) /(2 b)} f, \mathbb{R}^{n-1}\right\|_{[\gamma]}+\mid \Psi_{\rho, \theta}\left(D^{\prime}, D_{t}\right) f, \mathbb{R}^{n} \|_{[\gamma]}, & \theta \geq 0, \\ \left\|\Psi_{\rho, \sigma}\left(D^{\prime}, D_{t}\right) f, \mathbb{R}^{n}\right\|_{[\gamma]}, & \theta<0 .\end{cases}
$$

In this notation the estimate (6.9) can be rewritten as follows:

$$
\left\|\left(D_{n}^{\ell} f\right)(\cdot, 0, \cdot) ; \mathcal{H}^{r-\ell-1 / 2, s-\ell-1 / 2}\left(\mathbb{R}^{n}\right)\right\|_{[\gamma]} \leq c\left\|f ; \mathbb{R}^{n+1}\right\|_{[\gamma]}^{r, \frac{r}{2 b}, s} .
$$

\section{NONSTATIONARY PROBLEM}

We start with the a priori estimate in the half-space for solutions of the problem

$$
\begin{aligned}
A\left(D^{\prime}, D_{n}, D_{t}\right) u(x, t) & =f(x, t), \quad x_{n}>0, \\
\left(B_{j}\left(D^{\prime}, D_{n}, D_{t}\right) u\right)\left(x^{\prime}, 0, t\right) & =g_{j}\left(x^{\prime}, t\right), \quad j=1, \ldots, m,
\end{aligned}
$$


where $A(\xi, \tau)$ and $B_{j}(\xi, \tau)$ are quasihomogeneous polynomials in $\xi$ and $\tau$. We will look for estimates in the spaces $H_{[\gamma]+}^{r, \frac{r}{2 b}, s}\left(\mathbb{R}_{+}^{n+1}\right)$, where $r>s \geq 0$ and the conditions (5.2) are satisfied.

Definition 7.1. The problem (17.1) corresponding to the family of symbols $A(\xi, \tau)$, $B_{1}(\xi, \tau), \ldots, B_{m}(\xi, \tau)$ is called pseudoparabolic if the conditions (A), (B), $\left(\mathrm{C}_{1}\right),\left(\mathrm{C}_{2}\right)$, and $\left(\mathrm{C}_{3}\right)$ in the introduction are satisfied.

Let us note that the substitution $\tau \rightarrow \lambda^{2 b}$ transforms the family of symbols $A(\xi, \tau)$, $B_{1}(\xi, \tau), \ldots, B_{m}(\xi, \tau)$ satisfying the conditions of Definition 7.1 into the family of symbols $\tilde{A}(\xi, \lambda), \tilde{B}_{1}(\xi, \lambda), \ldots, \tilde{B}_{m}(\xi, \lambda)$, considered in $\S 3$ and corresponding to a weak parameter-elliptic problem.

Theorem 7.2. Let the problem (7.1) be pseudoparabolic and let the condition (5.5) be satisfied. Then

$$
\begin{aligned}
& \quad\left\|u ; \mathbb{R}_{+}^{n+1}\right\|_{[\gamma]}^{r, \frac{r}{2 b}, s} \leq \mathrm{const}\left({ }^{\prime}\left\|A\left(D_{x}, D_{t}\right) u ; \mathbb{R}_{+}^{n+1}\right\|_{[\gamma]}^{r-2 m, \frac{r-2 m}{2 b}, s-2 \mu}\right. \\
& \left.\quad+\sum_{j=1}^{m}\left\|\Psi_{r-b_{j}-1 / 2, s-\beta_{j}-1 / 2}\left(D_{x^{\prime}}, D_{t}\right)\left(B_{j}\left(D_{x}, D_{t}\right) u\right)(\cdot, 0, \cdot) ;\left(\mathbb{R}^{n}\right)\right\|_{[\gamma]}\right), \quad \gamma<0 .
\end{aligned}
$$

Using the Laplace transform in $\tau$, we reduce this theorem to Theorem 5.2, On the other hand, we can make the Fourier transform in $x^{\prime}$, the Laplace transform in $t$, and repeat the arguments in Theorem 5.2 .

In conclusion, we present a result about the solvability of the quasihomogeneous problem (7.1). The conditions imposed on the right-hand side were suggested by papers of S. A. Galpern on pseudohyperbolic equations (see a detailed discussion in [15] and 4]).

Theorem 7.3. Let the problem (7.1) be pseudoparabolic and let condition (5.5) be satisfied. Additionally, let the right-hand side $f \in H_{[\gamma]+}^{r-2 m, \frac{(r-2 m)}{(2 b)}, s-2 \mu}\left(\mathbb{R}_{+}^{n+1}\right)$ satisfy the conditions

$$
\begin{gathered}
\left(1+|x|^{2}\right)^{l / 2} f \in H_{[\gamma]+}^{r-2 m, \frac{(r-2 m)}{(2 b)}, s-2 \mu}\left(\mathbb{R}_{+}^{n+1}\right), \quad l>\frac{n}{2}+2 \mu, \\
\int_{\mathbb{R}^{n}} x^{\alpha} f(x, t) d x=0, \quad|\alpha| \leq 2 \mu-1 .
\end{gathered}
$$

Let the function $g_{j}\left(x^{\prime}, t\right)$ on the boundary satisfy the conditions

$$
\left\|\Psi_{r-b_{j}-1 / 2, s-\beta_{j}-1 / 2}\left(D_{x^{\prime}}, D_{t}\right) g_{j} ;\left(\mathbb{R}^{n}\right)\right\|_{[\gamma]}<\infty, \quad j=1, \ldots, m .
$$

Then there exists $\gamma_{0}<0$ such that for $\gamma<\gamma_{0}$ the problem (7.1) has a unique solution $u \in H_{[\gamma]+}^{r, \frac{r}{2 b}, s}\left(\mathbb{R}_{+}^{n+1}\right)$ and for this solution the following estimate holds:

$$
\begin{aligned}
& \quad\left\|u ; \mathbb{R}_{+}^{n+1}\right\|_{[\gamma]}^{r, \frac{r}{2 b}, s} \leq \mathrm{const}\left({ }^{\prime}\left\|f ; \mathbb{R}_{+}^{n+1}\right\|_{[\gamma]}^{r-2 m, \frac{(r-2 m)}{(2 b)}, s-2 \mu}\right. \\
& \left.\quad+\sum_{j=1}^{m}\left\|\Psi_{r-b_{j}-1 / 2, s-\beta_{j}-1 / 2}\left(D_{x^{\prime}}, D_{t}\right) g_{j} ;\left(\mathbb{R}^{n}\right)\right\|_{[\gamma]}\right), \quad \gamma<\gamma_{0} .
\end{aligned}
$$

Proof. 1. As in Theorem 5.2, we start with the homogeneous problem, where $f \equiv 0$. After the Fourier-Laplace transform in the variables $\left(x^{\prime}, t\right)$ we obtain the following problem on the half-line:

$$
\begin{aligned}
A\left(\xi^{\prime}, D_{n}, \tau\right) \hat{u}^{\prime}\left(\xi^{\prime}, x_{n}, \tau\right) & =0, \quad x_{n}>0, \\
\left(B_{j}\left(\xi^{\prime}, D_{n}, \tau\right) \hat{u}^{\prime}\right)\left(\xi^{\prime}, 0, \tau\right) & =\hat{g}_{j}\left(\xi^{\prime}, \tau\right), \quad j=1, \ldots, m,
\end{aligned}
$$


and the solution of this problem (cf. the proof of Theorem 5.2) is written in the form

$$
\hat{u}^{\prime}\left(\xi^{\prime}, x_{n}, \tau\right)=\sum_{j=1}^{m} \hat{g}_{j}\left(\xi^{\prime}, \tau\right) v_{j}\left(\xi^{\prime}, x_{n}, \tau\right),
$$

where $v_{j}\left(\xi^{\prime}, x_{n}, \tau\right), j=1, \ldots, m$, is the family of fundamental solutions of the ordinary differential operator $A\left(\xi^{\prime}, D_{n}, \tau\right)$. According to Theorem 3.2 there is $\gamma_{0}<0$ such that the functions $v_{j}\left(\xi^{\prime}, x_{n}, \tau\right)$ are holomorphic in $\tau$ for $\operatorname{Im} \tau<\gamma_{0}$. Repeating the arguments used in the proof of Proposition 5.3 one can prove that

$$
\begin{gathered}
\left(\int_{0}^{\infty}\left|D_{n}^{\ell} v_{j}\left(x_{n}, \xi^{\prime}, \tau\right)\right|^{2} d x_{n}\right)^{1 / 2} \leq \mathrm{const} \frac{\left|\Psi_{r-b_{j}-1 / 2, s-\beta_{j}-1 / 2}\left(\xi^{\prime}, \tau\right)\right|}{\left|\Psi_{r-\ell, s-\ell}\left(\xi^{\prime}, \tau\right)\right|} \\
l=0,1, \ldots, r, \quad \operatorname{Im} \tau<\gamma_{0} .
\end{gathered}
$$

Therefore (cf. estimates (5.100)

$$
\begin{aligned}
& \int_{\mathbb{R}^{n-1}}\left|\Psi_{r-\ell, s-\ell}\left(\xi^{\prime}, \tau\right)\right|^{2} \int_{0}^{\infty}\left|D_{n}^{\ell} \hat{u}^{\prime}\left(\xi^{\prime}, x_{n}\right)\right|^{2} d x_{n} d \xi^{\prime} \\
& \quad \leq \operatorname{const} \sum_{j=1}^{m} \int_{\mathbb{R}^{n-1}}\left|\Psi_{r-b_{j}-1 / 2, s-\beta_{j}-1 / 2}\left(\xi^{\prime}, \tau\right) g_{j}^{\prime}\left(\xi^{\prime}, \tau\right)\right|^{2} d \xi^{\prime} .
\end{aligned}
$$

According to (7.5), the right-hand side is uniformly bounded for $\operatorname{Im} \tau<\gamma_{0}$. Adding up the left-hand sides for $l$ between 0 and $r$, we obtain on the left a norm that is equivalent to the norm in $H_{[\rho]}^{r, \frac{r}{2 b}, s}\left(\mathbb{R}_{+}^{n+1}\right)$ for $\rho=\operatorname{Im} \tau$. Therefore,

$$
\sup _{\rho<\gamma}\left\|u ; \mathbb{R}_{+}^{n+1}\right\|_{[\rho]}^{r, \frac{r}{2 b}, s}<\infty, \quad \gamma<\gamma_{0} .
$$

By the Paley-Wiener theorem (Theorem 6.2),$u \in H_{[\gamma]+}^{r, \frac{r}{2 b}, s}\left(\mathbb{R}_{+}^{n+1}\right)$.

2. Now, similarly to Theorem 5.2, we reduce the nonhomogeneous problem to a homogeneous one using a solution of the nonhomogeneous equation.

Proposition 7.4. Let a quasihomogeneous polynomial $A(\xi, \tau)$ be pseudoparabolic and let the right-hand side $f \in H_{[\gamma]+}^{r-2 m, \frac{(r-2 m)}{(2 b)}, s-2 \mu}\left(\mathbb{R}_{+}^{n+1}\right)$ of equation (17.1) satisfy conditions (7.3) and (7.4). Then there exists a function $v \in H_{[\gamma]+}^{r, \frac{r}{2 b}, s}\left(\mathbb{R}_{+}^{n+1}\right)$ satisfying the equation

$$
A\left(D_{x}, D_{t}\right) v(x, t)=f(x, t), \quad x_{n}>0,
$$

and such that

$$
\begin{gathered}
\|\left(\Psi_{r-b_{j}-1 / 2, s-\beta_{j}-1 / 2}\left(D_{x^{\prime}}, D_{t}\right)\left(B_{j}\left(D_{x}, D_{t}\right) v\right)(\cdot, 0, \cdot) ;\left(\mathbb{R}^{n}\right) \|_{[\gamma]}\right. \\
\quad \leq \mathrm{const}\left\|f ; \mathbb{R}_{+}^{n+1}\right\|_{[\gamma]}^{r-2 m, \frac{(r-2 m)}{(2 b)}, s-2 \mu}, \quad j=1, \ldots, m .
\end{gathered}
$$

Proof. The proof of the proposition is based on the following simple fact.

Lemma 7.5. Let a function $h(x)$ be in the space $H^{k}\left(\mathbb{R}^{n}\right)$ and satisfy the conditions

$$
\left(1+|x|^{2}\right)^{l / 2} h \in H^{k}\left(\mathbb{R}^{n}\right), \quad l>\frac{n}{2}+\nu, \quad \int_{\mathbb{R}^{n}} x^{\alpha} h(x) d x=0, \quad|\alpha| \leq \nu-1 .
$$

Then the function $h(x)$ can be represented in the form

$$
h(x)=\left|D_{x}\right|^{\nu} g(x), \quad g(x) \in H^{k+\nu}\left(\mathbb{R}^{n}\right), \quad\|g\|^{k+\nu} \leq \mathrm{const}\|h\|^{k} .
$$

Proof. By the hypotheses of the lemma the Fourier transform $\hat{h}(\xi)$ is in the space $C^{\nu}$ of $\mu$ times continuously differentiable functions and at the point $\xi=0$ it has a zero of order $\nu$. Denoting by $g(x)$ the inverse Fourier transform of the function $|\xi|^{-n u} \hat{h}(\xi)$ we obtain the statement of the lemma. (For more details, see [15, Proposition 5.2].) 
Now we prove Proposition 7.4. Let $f^{*}$ be an extension of the function $f$ to the entire space $\mathbb{R}^{n+1}$. Using the Hestenes extension one can ensure that

$$
f^{*} \in H_{[\gamma]+}^{r-2 m, \frac{(r-2 m)}{(2 b)}, s-2 \mu}\left(\mathbb{R}^{n+1}\right)
$$

and

$$
\left\|f^{*} ; \mathbb{R}^{n+1}\right\|_{[\gamma]}^{r-2 m, \frac{(r-2 m)}{(2 b)}, s-2 \mu} \leq \mathrm{const}\left\|f ; \mathbb{R}_{+}^{n+1}\right\|_{[\gamma]}^{r-2 m, \frac{(r-2 m)}{(2 b)}, s-2 \mu} .
$$

Moreover, for the function $f^{*}$, conditions (7.3) and (7.4) are satisfied. According to Lemma 7.5 we can find a function $g^{*} \in H_{[\gamma]+}^{r-2 m+2 \mu, \frac{(r-2 m+2 \mu)}{(2 b)}, s}\left(\mathbb{R}^{n+1}\right)$ such that $f^{*}=$ $\left|D_{x}\right|^{2 \mu} g^{*}$ and

$$
\left\|g^{*} ; \mathbb{R}^{n+1}\right\|_{[\gamma]}^{r-2 m+2 \mu, \frac{(r-2 m+2 \mu)}{(2 b)}, s} \leq \mathrm{const}\left|f ; \mathbb{R}_{+}^{n+1}\right|_{[\gamma]}^{r-2 m, \frac{(r-2 m)}{(2 b)}, s-2 \mu} .
$$

Now we set

$$
v^{*}(x, t)=A^{-1}\left(D_{x}, D_{t}\right) f^{*}(x, t)=\left|D_{x}\right|^{2 \mu} A^{-1}\left(D_{x}, D_{t}\right) g^{*}(x, t) .
$$

Then $v^{*} \in H_{[\gamma]+}^{r, \frac{r}{2 b}, s}\left(\mathbb{R}^{n+1}\right)$.

Denote by $v$ the restriction of the function $v^{*}$ to $\mathbb{R}_{+}^{n+1}$. Then $v \in H_{[\gamma]+}^{r, \frac{r}{2 b}, s}\left(\mathbb{R}_{+}^{n+1}\right)$ and $\left\|v ; \mathbb{R}_{+}^{n+1}\right\|_{[\gamma]}^{r, \frac{r}{2 b}, s} \leq$ const $\left\|f ; \mathbb{R}_{+}^{n+1}\right\|_{[\gamma]}^{r-2 m, \frac{(r-2 m)}{(2 b)}, s-2 \mu}$. It is clear that (17.9) holds.

Next, the operator

$$
B_{j}\left(D_{x}, D_{t}\right): H_{[\gamma]+}^{r, \frac{r}{2 b}, s}\left(\mathbb{R}_{+}^{n+1}\right) \rightarrow H_{[\gamma]+}^{r-b_{j}, \frac{\left(r-b_{j}\right)}{(2 b)}, s-\beta_{j}}\left(\mathbb{R}_{+}^{n+1}\right)
$$

is continuous. Since the trace of a function in

$$
H_{[\gamma]+}^{r-b_{j}, \frac{\left(r-b_{j}-(1 / 2) \beta_{j}\right)}{(2 b)}, s-\beta_{j}}\left(\mathbb{R}_{+}^{n+1}\right)
$$

belongs to

$$
H_{[\gamma]+}^{r-b_{j}-1 / 2, \frac{\left(r-b_{j}-(1 / 2) \beta_{j}\right)}{(2 b)}, s-\beta_{j}-1 / 2}\left(\mathbb{R}^{n}\right),
$$

we get (7.10).

3. Let us complete the proof of Theorem 7.3 We will look for a solution in the form

$$
u(x, t)=v(x, t)+w(x, t),
$$

where $v(x, t)$ is the function from Proposition [7.4. Then $w(x, t)$ is a solution of the problem

$$
\begin{gathered}
A\left(D_{x}, D_{t}\right) w(x, t)=0, \quad x_{n}>0, \\
\left(B_{j}\left(D x, D_{t}\right) u\right)\left(x^{\prime}, 0, t\right)=g_{j}\left(x^{\prime}, t\right)-\left(B_{j}\left(D x, D_{t}\right) v\right)\left(x^{\prime}, 0, t\right), \quad j=1, \ldots, m .
\end{gathered}
$$

According to Proposition 7.4 the boundary values in (7.12) belong to

$$
H_{[\gamma]+}^{r-b_{j}-1 / 2, \frac{\left(r-b_{j}-(1 / 2) \beta_{j}\right)}{(2 b)}, s-\beta_{j}-1 / 2}\left(\mathbb{R}^{n}\right) .
$$

By what we have already proved, the solution $w(x, t)$ exists and belongs to the space $H_{[\gamma]+}^{r, \frac{r}{2 b}, s}\left(\mathbb{R}_{+}^{n+1}\right)$. Theorem 7.3 is proved. 


\section{REFERENCES}

[1] M. I. Vishik and L. A. Lyusternik, Regular degeneration and boundary layer for linear differential equations with small parameter. Uspekhi Mat. Nauk 12 (1957), no. 5, 3-122; English transl., Amer. Math. Soc. Transl. (2), vol. 20, Amer. Math. Soc., New York, 1962. pp. 239-364. MR0136861 $(25: 322)$

[2] L. R. Volevich, The Vishik-Lyusternik method in elliptic problems with a small parameter. Tr. Mosk. Mat. Obs. 67 (2006), 104-147; English transl., Trans. Moscow Math. Soc., 2006, 87-126. MR 2301592

[3] M. S. Agranovich and M. I. Vishik, Elliptic problems with a parameter and parabolic problems of general type. Uspekhi Mat. Nauk 19 (1964) no. 3, 53-161. (Russian) MR0192188 (33:415)

[4] G. V. Demidenko and S. V. Uspenskiur, Equations and systems unresolved with respect to the highest derivative. "Nauchnaya Kniga", Novosibirsk, 1968; English transl., Marcel Dekker, New York, 2003. MR2064818 (2005a:35001)

[5] R. Denk, R. Mennicken, and L. R. Volevich, Boundary value problems for a class of elliptic operator pencils. Integ. Eq. Operator Th. 8 (2000), 410-436. MR 1799649(2001k:35128)

[6] _ On elliptic operator pencils with general boundary conditions. Integ. Eq. Operator Th. 9 (2001), 25-40. MR 1806842(2002a:47020)

[7] S. A. Nazarov, The Vishik-Lyusternik method for elliptic boundary value problems in regions with conic points. I. The problem in a cone, Sibirsk. Mat. Zh. 22 (1981), 142-163, English transl., Siberian Math. J. 22 (1982), 594-611. MR624412 (83m:35046a)

[8] L. R. Volevich and B. P. Paneah, Some spaces of generalized functions and embedding theorems. Uspekhi Mat. Nauk 20 (1965), 3-74; English transl., Russian Math. Surv. 20 (1965), 1-73. MR0174970 $(30: 5160)$

[9] S. A. Nazarov, On the index of the boundary problem with a small parameter at the highest derivatives. Differential'nye Uravneniya i ih Primeneniya, vyp. 24, Vilnius, 1979, pp. 75-84. (Russian) MR566964 (82f:35072)

[10] R. C. Gunning and H. Rossi, Analytic functions of several complex variables. Prentice-Hall, Englewood Cliffs, NJ, 1965. MR0180696 (31:4927)

[11] S. G. Gindikin and L. R. Volevich, Mixed problem for partial differential equations with quasihomogeneous principal part. Amer. Math. Soc., Providence, RI, 1996. MR.1357662 (96j:35145)

[12] L. S. Frank, Coercive singular perturbations. I. A priori estimates. Annali Mat. Pura Appl. 119 (1979), 41-113. MR551218 (81b:35025)

[13] S. Agmon, A. Douglis, and L. Nirenberg, Estimates near the boundary for solutions of elliptic partial differential equations satisfying general boundary conditions. I. Comm. Pure Appl. Math. 12 (1959). 623-727. MR0125307 (23:A2610)

[14] S. G. Gindikin and L. R. Volevich, Distributions and convolution equations. Gordon and Breach, Philadelphia, PA, 1992. MR1155843 (93c:46061)

[15] I. Fedotov and L. R. Volevich. The Cauchy problem for hyperbolic equations not resolved with respect to the highest time derivative. Russian J. Math. Phys. 13 (2006), no. 3, 278-292. MR2262830

Keldysh Institute of Applied Mathematics, Russian Academy of Sciences, 4 Miusskaya Place, Moscow 125047, Russia

Translated by O. A. KHLEBORODOVA 